\title{
Synthetic observations of spiral arm tracers of a simulated Milky Way analog
}

\author{
S. Reissl ${ }^{1}$, J. M. Stil ${ }^{2}$, E. Chen ${ }^{3}$, R. G. Treß ${ }^{1}$, M. C. Sormani ${ }^{1}$, R. J. Smith ${ }^{3}$, R. S. Klessen ${ }^{1,4}$, M. Buick ${ }^{2}$, \\ S. C. O. Glover ${ }^{1}$, R. Shanahan ${ }^{2}$, S. J. Lemmer ${ }^{2}$, J. D. Soler ${ }^{5}$, H. Beuther ${ }^{5}$, J. S. Urquhart ${ }^{6}$, L. D. Anderson ${ }^{7,8,9}$, \\ K. M. Menten ${ }^{10}$, A. Brunthaler ${ }^{10}$, S. Ragan ${ }^{11}$, and M. R. Rugel ${ }^{10}$ \\ ${ }^{1}$ Universität Heidelberg, Zentrum für Astrononmie, Institut für Theoretische Astrophysik, Albert-Ueberle-Str. 2, 69120 Heidelberg, \\ Germany \\ e-mail: reissl@uni-heidelberg.de \\ 2 Department of Physics and Astronomy, The University of Calgary, 2500 University Drive NW, Calgary AB T2N 1N4, Canada \\ ${ }^{3}$ Jodrell Bank Centre for Astrophysics, School of Physics and Astronomy, University of Manchester, Oxford Road, Manchester, M13 \\ 9PL, UK \\ ${ }^{4}$ Universität Heidelberg, Interdisziplinäres Zentrum für Wissenschaftliches Rechnen, Im Neuenheimer Feld 205, 69120 Heidelberg, \\ Germany \\ ${ }^{5}$ Max Planck Institute for Astronomy, Königstuhl 17, 69117 Heidelberg, Germany \\ ${ }^{6}$ Centre for Astrophysics and Planetary Science, University of Kent, Canterbury CT2 7NH, UK \\ ${ }^{7}$ Department of Physics and Astronomy, West Virginia University, Morgantown, WV 26506, USA \\ 8 Adjunct Astronomer at the Green Bank Observatory, PO Box 2, Green Bank, WV 24944, USA \\ ${ }^{9}$ Center for Gravitational Waves and Cosmology, West Virginia University, Chestnut Ridge Research Building, Morgantown, \\ WV 26505, USA \\ ${ }^{10}$ Max-Planck-Institut für Radioastronomie, Auf dem Hügel 69, 53121 Bonn, Germany \\ ${ }^{11}$ School of Physics \& Astronomy, Cardiff University, The Parade, Cardiff CF24 3AA, UK
}

Received 8 February 2020 / Accepted 20 July 2020

\begin{abstract}
Context. The Faraday rotation measure (RM) is often used to study the magnetic field strength and orientation within the ionized medium of the Milky Way. Recent observations indicate an RM magnitude in the spiral arms that exceeds the commonly assumed range. This raises the question of how and under what conditions spiral arms create such strong Faraday rotation.

Aims. We investigate the effect of spiral arms on Galactic Faraday rotation through shock compression of the interstellar medium. It has recently been suggested that the Sagittarius spiral arm creates a strong peak in Faraday rotation where the line of sight is tangent to the arm, and that enhanced Faraday rotation follows along side lines which intersect the arm. Here our aim is to understand the physical conditions that may give rise to this effect and the role of viewing geometry.

Methods. We apply a magnetohydrodynamic simulation of the multi-phase interstellar medium in a Milky Way-type spiral galaxy disk in combination with radiative transfer in order to evaluate different tracers of spiral arm structures. For observers embedded in the disk, dust intensity, synchrotron emission, and the kinematics of molecular gas observations are derived to identify which spiral arm tangents are observable. Faraday rotation measures are calculated through the disk and evaluated in the context of different observer positions. The observer's perspectives are related to the parameters of the local bubbles surrounding the observer and their contribution to the total Faraday rotation measure along the line of sight.

Results. We reproduce a scattering of tangent points for the different tracers of about $6^{\circ}$ per spiral arm similar to the Milky Way. For the RM, the model shows that compression of the interstellar medium and associated amplification of the magnetic field in spiral arms enhances Faraday rotation by a few hundred $\mathrm{rad} \mathrm{m}^{-2}$ in addition to the mean contribution of the disk. The arm-interarm contrast in Faraday rotation per unit distance along the line of sight is approximately $\sim 10$ in the inner Galaxy, fading to $\sim 2$ in the outer Galaxy in tandem with the waning contrast of other tracers of spiral arms. We identify a shark fin pattern in the RM Milky Way observations and in the synthetic data that is characteristic for a galaxy with spiral arms.
\end{abstract}

Key words. Galaxy: structure - Galaxy: fundamental parameters - ISM: bubbles - ISM: magnetic fields - local insterstellar matter

\section{Introduction}

The magnetic field of the Milky Way affects the physics of the interstellar medium on various scales (Klessen \& Glover 2016). In turn, the magnetic field is shaped and amplified by motions in the interstellar medium (Beck 2015), including turbulent motions injected by stellar winds and supernova explosions (Ferriere et al. 1991; Tomisaka 1998), and by streaming motions of gas. In particular, spiral arms can shape the magnetic field on a galactic scale as the gas entering the arms is compressed in a shock (Roberts 1969). The strength and the structure of the magnetic field in spiral arms are important initial conditions for theories of star formation, cosmic ray diffusion, and dynamics of the interstellar medium, for example the formation of filaments (Inoue et al. 2018) and the Parker instability (Körtgen et al. 2018).

The detection of spiral arms within the Milky Way is aggravated by the fact that the view toward the Milky Way's spiral arms and the Galactic center is naturally blocked. Due to the 
position of our own Solar System within the Galactic disk dust extinction at optical and UV wavelengths are hampered. However, observations of synchrotron radiation and far-infrared dust emission (Beuermann et al. 1985; Beuther et al. 2012), very long baseline interferometry (VLBI) parallaxes of maser sources associated with high-mass star-forming regions (Reid et al. 2019), and Faraday rotation measurements (Shanahan et al. 2019) open a window to infer the spiral structure of our home galaxy. In detail, the observations of diffuse synchrotron emission in wellresolved face-on galaxies show evidence for amplified ordered magnetic fields associated with tracers of spiral arm shocks (e.g., Jansson \& Farrar 2012). The polarized intensity shows ordering of the magnetic field in the plane of the sky, in a direction perpendicular to the plane of polarization. This fact cannot be used to prove the presence of large-scale magnetic fields, because the polarized signal of a compressed turbulent magnetic field is the same as that of a turbulent field superposed on a large-scale magnetic field (Sokoloff et al. 1998). Faraday rotation in turn can reveal large-scale magnetic fields (Berkhuisen et al. 2003; Gaensler et al. 2005) and magnetic field reversals (e.g., SimardNormandin \& Kronberg 1979; Mora-Partiarroyo et al. 2019).

The large-scale magnetic field of the Milky Way can be studied in much greater detail with all-sky dust polarimetry (e.g., Planck Collaboration Int. XIX 2015; Planck Collaboration XII 2020). Further details about the Milky Way's magnetic field can be gathered by Faraday rotation observations of pulsars and polarized extragalactic radio sources (Han et al. 2006; Jansson \& Farrar 2012). These Faraday rotation observations reveal that the magnetic field in the Sagittarius arm is reversed with respect to the main magnetic field direction in the inner Galaxy. The existence of other magnetic field reversals is the subject of continued debate.

Recently, Shanahan et al. (2019) presented a pattern of Faraday rotation along the Galactic plane based on data provided by the THOR survey (see Beuther et al. 2016; Wang et al. 2020, for details). Here Shanahan et al. (2019) suggests that the Sagittarius arm is a much stronger Faraday screen within the Milky Way than previously thought. This pattern consists of a strong peak in Faraday rotation associated with the spiral arm tangent, flanked by enhanced but lower Faraday rotation along lines of sight through the inner Galaxy that intersect the arm, and much less Faraday rotation along lines of sight beyond the tangent that do not intersect the arm.

Further insight into the magnetic field structure of spiral arms can be obtained if the observed pattern can be reproduced by numerical models of the flow of magnetized interstellar medium through the spiral arms (Fletcher et al. 2011). Such simulations can provide insight into the importance of viewing geometry, while connecting key physics to observable quantities without the need to deproject the observed quantities. This requires implementation of emission and radiation transfer based on local conditions defined by the simulation. Direct comparison of magnetohydrodynamic (MHD) simulations with Faraday rotation data on a galactic scale are exceedingly rare, while a some studies have addressed MHD turbulence without Galactic structure (e.g., super bubbles Pakmor et al. 2018).

What is required to relate the simulations to observations of Galactic Faraday rotation? The main observed properties are the magnitude and sign of Faraday rotation in relation to other tracers of spiral arms from the perspective of an observer embedded in the disk of the Milky Way. Simulations must therefore reproduce the 3D structure of the multi-phase interstellar medium with a magnetic field, and generate mock observations of key tracers such as dust and synchrotron emission of the multi-phase interstellar medium that can be compared with the Milky Way in a statistical sense. In the Milky Way, spiral arms are best traced by kinematics of the molecular and atomic gas, while directions of spiral arm tangents are also traced by excess emission in the form of radio continuum emission from synchrotron radiation and free-free emission from star-forming regions (e.g., Beuermann et al. 1985), and dust emission (e.g., Beuther et al. 2012). Self-consistent treatment of these observables is key to effectively relating simulations to observations.

The extent to which the signatures of large-scale Galactic structure stand out among other effects, such as the structure of the local interstellar medium, is another question that may be addressed with simulations. Mock observations generated by radiative transfer post-processing codes can be evaluated from positions in the simulations that best represent the perspective of an observer on Earth. In this paper, we bridge the gap between simulations of gas dynamics and magnetic field evolution on a galactic scale with observations of Milky Way-like spiral structures through the implementation of emission processes and radiation transfer through ray tracing. However, we note that key aspects of such a complex system like the Milky Way are still a field of ongoing research. Naturally, any Milky Way analog may only cover certain physical processes and remains merely an approximation. The resulting synthetic observations can rarely directly be compared with the original, and should be taken with care.

This paper is structured as follows. In Sect. 2 we summarize our numerical model of the Galactic disk with spiral arms and the physical effects that it includes. In Sect. 3 we outline the radiative transfer post-processing techniques and the basic physical properties of the different tracers for which synthetic data is generated in this paper. We present the synthetic observations in Sect. 4, and analyze their longitudinal profiles to determine the tangent points of the spiral arms. We temporarily change the perspective to that of an outside observer in Sect. 5.1 in order to evaluate the validity of our spiral arm detections. In Sect. 5.2 we go back to the perspective of an observer within the Galactic disk and explore the possibility of deriving the pitch angle of our Local Arm in the Milky Way by means of Faraday RM observations. In Sect. 5.3 we offer a purely geometric explanation for the characteristic shark fin peak profile in the longitudinal profiles. This profile constitutes the observational signature of Faraday rotation at a spiral arm tangent point. In Sect. 5.4 we investigate where Faraday rotation originates along the line of sight by tracing the propagation of polarized radio waves from the outside of the model Galaxy toward the observer. In Sect. 6 we investigate the implications that the existence of a local bubble has for the detection of the spiral arms. In Sect. 7 we discuss the impact that the simplifications made in our numerical model may have on our results. Finally, we summarize our results in Sect. 8 .

\section{The Milky Way model}

In this section we outline the numerical methods and the physical principles for constructing our Milky Way-disk analog.

\subsection{Numerical methods}

We perform a galactic-scale MHD simulation using the movingmesh code AREPO (Springel 2010) to model the magnetized interstellar medium (ISM). The simulations include gas selfgravity, magnetic fields, a live chemical network, sink particles to represent star formation, supernova (SN) feedback, and an externally imposed four-armed spiral potential. 


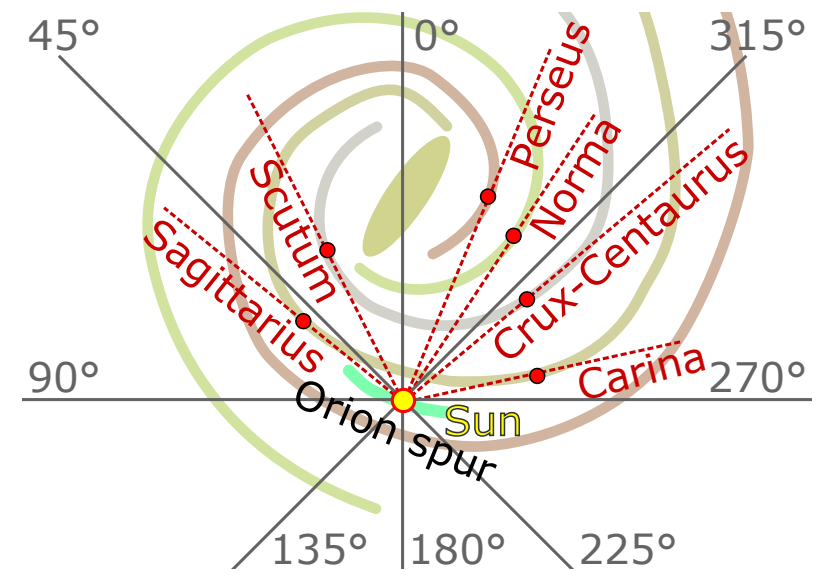

Fig. 1. Sketch of the Milky Way's spiral structure. The spiral arms are indicated in separate colors. Our Solar System is placed in the Orion spur about $8.2 \mathrm{kpc}$ away from the Galactic center. Together with the Milky Way's center, the Sun defines the Galactic coordinate system where angles are counted counterclockwise along the longitude.

This galaxy model forms part of the "Cloud Factory" simulation suite recently presented in Smith et al. (2020), which aims to investigate the connection between galactic-scale forces and star formation in individual molecular clouds. We employ an idealized externally imposed galactic potential that consists of an axisymmetric part plus a spiral perturbation. The axisymmetric part is composed of the sum of a stellar disk, bulge and dark matter halo according to the best fitting model of McMillan (2017). A spiral perturbation as introduced by Cox \& Gomez (2002) is added to this potential to give four well-defined spiral arms resembling the Milky Way (compare Fig. 1 and Reid et al. 2019). The spiral potential has a pitch angle ${ }^{1} \Psi=15^{\circ}$ and a pattern speed of $2 \times 10^{-8} \mathrm{rad} \mathrm{yr}^{-1}$, which corresponds to a co-rotation radius of $11 \mathrm{kpc}$ (see also Smith et al. 2014).

The ISM is assumed to be composed of atomic hydrogen and helium, together with carbon and oxygen, with abundances matching those in the local ISM. The chemical evolution of the gas is followed using the NL97 chemical network of Glover \& Clark (2012), which has been extensively used in previous numerical studies of the ISM (see, e.g., Glover \& Clark 2012; Smith et al. 2014; Smith et al. 2020; Walch et al. 2015; Sormani et al. 2018; Tress et al. 2020). This network combines the treatment of hydrogen chemistry introduced in Glover \& Mac Low (2007) with a simplified model for CO formation and destruction taken from Nelson \& Langer (1997). The NL97 network includes $\mathrm{H}_{2}$ formation on grains, $\mathrm{H}_{2}$ photodissociation, collisional dissociation, $\mathrm{H}^{+}$recombination on grains and in the gas phase (see Table 1 of Glover \& Mac Low 2007), a highly approximate treatment of $\mathrm{CO}$ formation, $\mathrm{CO}$ photodissociation, and cosmic ray ionization of $\mathrm{H}$ and $\mathrm{H}_{2}$. The only charged species traced in the NL97 network are $\mathrm{H}^{+}$and $\mathrm{C}^{+}$, and so the density of free electrons is given by $n_{\mathrm{el}}=n_{\mathrm{H}^{+}}+n_{\mathrm{C}^{+}}$. A full description of the NL97 network can be found in Glover \& Clark (2012).

For simplicity, we assume a uniform interstellar radiation field (ISRF), with the intensity and spectrum given by Draine (1978). The attenuation of this radiation field in dense molecular clouds owing to dust shielding and $\mathrm{H}_{2}$ self-shielding is modeled using the TreeCol algorithm of Clark et al. (2012). The cosmic

\footnotetext{
1 The pitch angle is sometimes defined in the literature with respect to the magnetic field direction. Here the pitch angle is the deviation of the spiral arm with respect to a perfect circle around the Galactic center (i.e., $\Psi=0^{\circ}$ ).
}

ray ionization rate is also assumed to be spatially constant, with a value of $\zeta_{\mathrm{H}}=3 \times 10^{-17} \mathrm{~s}^{-1}$ for atomic hydrogen. Ionization rates for other chemical species are scaled from this value as described in Glover \& Clark (2012).

Star formation is represented by non-gaseous sink particles, which are formed from gravitationally bound gas above a threshold gas density of $n_{\mathrm{g}}=10^{3} \mathrm{~cm}^{-3}$. The sinks can accrete gas and form stars with a star formation efficiency of $2 \%$, which is consistent with observations in giant molecular clouds (GMCs) in the Milky Way (e.g., Krumholz \& Tan 2007). The sinks represent small clusters of stars (the maximum stellar content of the sinks is set to $200 M_{\odot}$ in this run) and have SN feedback tied to them as outlined in Tress et al. (2020). Here we also include a random SN component in the simulations to represent Type Ia supernovae. Full details of the numerical methods we use can be found in Smith et al. (2020). However, Smith et al. (2020) present only hydrodynamic models. The models used in this paper include ideal MHD using the setup applied in the follow-up paper by Chen et al. (in prep.). To do this we use the MHD capabilities of AREPO, as described in Pakmor \& Springel (2013), which uses the Powell divergence control scheme (Powell et al. 1999). This methodology has previously been successfully used to model galactic-scale magnetic fields in Pakmor et al. (2017).

\subsection{The simulation setup}

The initial setup is generated using the following procedure. We assign disk gas densities according to the HI thin and thick disk components of McMillan (2017) to be consistent with our analytic potential. The disk is truncated at a radius of $15 \mathrm{kpc}$ and surrounded by a hot diffuse medium for reasons of computational efficiency. We allocate the initial velocity for every gas cell following the rotation curve that arises from our analytic potential $\left(0 \mathrm{~km} \mathrm{~s}^{-1}\right.$ at the galactic center, and $220 \mathrm{~km} \mathrm{~s}^{-1}$ from 4 kpc outward).

The run has two phases. Initially, for the first $200 \mathrm{Myr}$, we switch off gas self-gravity and the sink particles and SN feedback modules, but include the galactic potential. Doing this allows the galaxy to become relaxed, the field to undergo an initial amplification, and the spiral arms to be generated. The strength of the seed field is set to the commonly assumed value of $0.04 \mu \mathrm{G}$ (e.g., Pakmor et al. 2017) in a toroidal fashion. During this period we set the resolution refinement criteria for the Voronoi cells to a target mass of $10^{4} M_{\odot}$.

After $200 \mathrm{Myr}$, when the simulations reach a quasi-steady state, we turn on self-gravity, together with the sink particles and $\mathrm{SN}$ feedback modules, to allow the gas to collapse to form stars and then be disrupted by feedback. We run with this physics for a further $50 \mathrm{Myr}$ and use a higher resolution target mass with a maximum of $10^{3} M_{\odot}$. We also require that the Jeans length (Jeans 1902) is resolved by a minimum of four Voronoi cells necessary to avoid artificial fragmentation (Truelove et al. 1997). In this way we reach much higher mass resolution at GMC scales. The typical spatial resolution in this phase varies with the gas density, but for gas at $n_{\mathrm{g}} \sim 100 \mathrm{~cm}^{-3}$ it corresponds to a cell size of about $L \approx 1 \mathrm{pc}$. All our following analysis is performed using data from a snapshot produced after a total simulation time of $250 \mathrm{Myr}$ (i.e., $50 \mathrm{Myr}$ after we turn on self-gravity).

Observational tracers such as dust and synchrotron emission are highly sensitive to the local conditions (Reissl et al. 2019). For the purpose of the follow-up radiative transfer postprocessing we manually added three additional bubbles driven by clustered supernovae chosen to be roughly at solar distances from the Galactic center, each associated with a spiral arm. At 

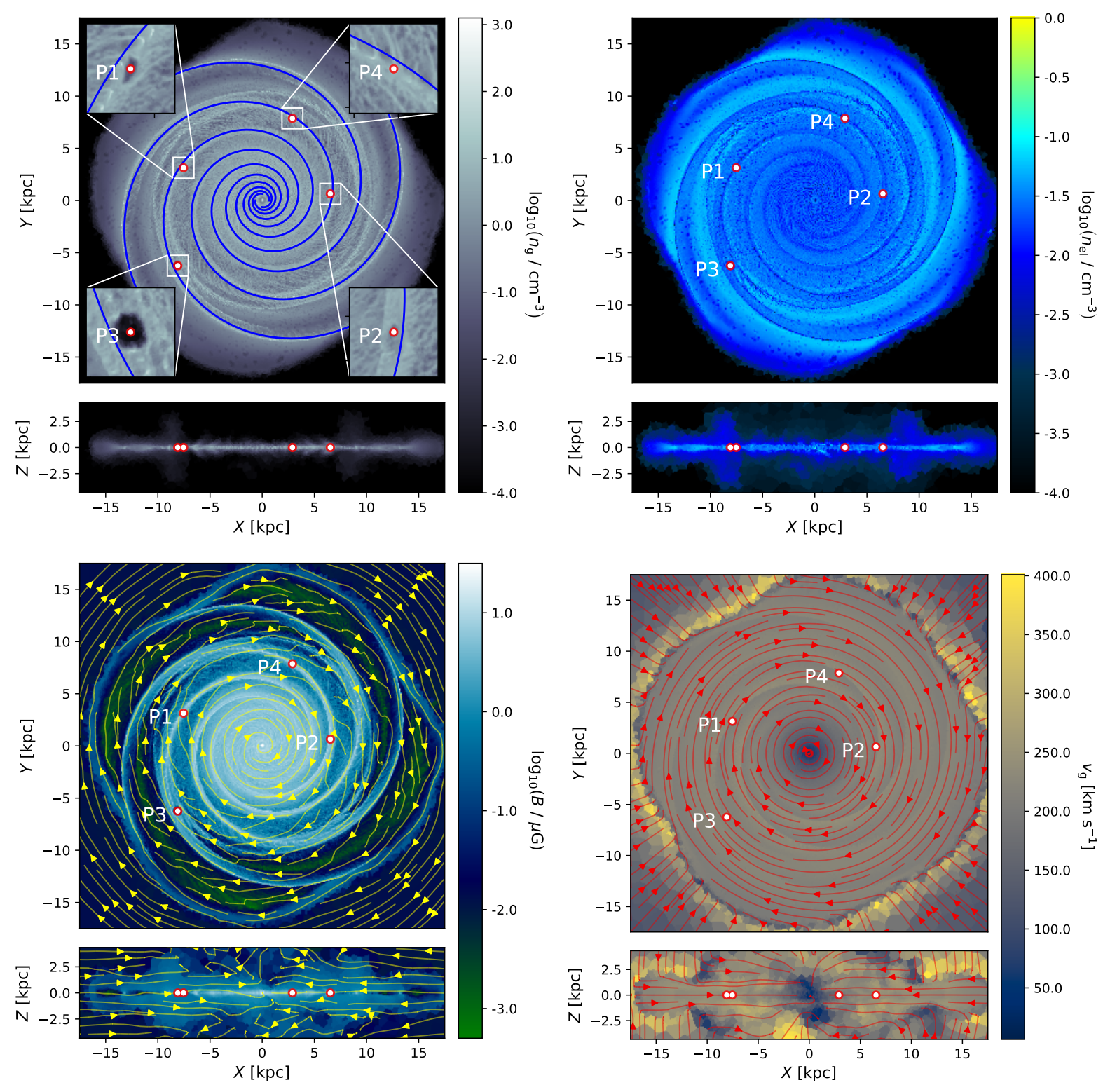

Fig. 2. Slices though the Milky Way model described in Sect. 2.2. Clockwise from top left to bottom right: gas density $n_{\mathrm{g}}$, electron density $n_{\mathrm{el}}$, velocity $v_{\mathrm{g}}$, and magnetic field strength $B$. The red circles and white labels indicate the observer positions considered in the paper. The insets in the $n_{\mathrm{g}}$ map (top left) show the local environment for each of the observers P1, P2, P3, and P4, and the blue spirals indicate the potential of our model. The yellow and red vector fields show the orientation of the magnetic field (bottom left) and the velocity field (bottom right).

these locations at $t=225 \mathrm{Myr}$ the number of $\mathrm{SNe}$ associated with a local sink particle is increased to 500. The SNe then explode with a roughly even distribution in time within the following $10 \mathrm{Myr}$, leading to density cavities representing different stages of bubble evolution. We place three observers at positions within these SN bubbles. Additionally, we choose an observer to be within a spiral arm region largely unaffected by any SN feedback for later analysis and comparison. Hereafter these observer positions are denoted P1, P2, P3, and P4 and have a distance of $8.16,6.50,10.22$, and $8.38 \mathrm{kpc}$, respectively, from the Galactic center (see Fig. 2). A detailed description and analysis of the impact of these locations with respect to observations is provided in Sect. 6.

In Fig. 2 we present cuts through to the disk midplane for the selected snapshot of the MHD simulation at $t=250 \mathrm{Myr}$. The cut of the gas number density distribution $n_{\mathrm{g}}$ shows the four welldefined spiral arms. The arms blend into a diffuse disk in the center since we are lacking a proper bulge treatment. However, this does not affect the RT modeling and analysis performed in this paper since our focus is on spiral arm detections in the outer regions. The electron distribution $n_{\mathrm{el}}$ has a pattern similar to that of the gas density with values comparable to other models of the Milky Way (Cordes \& Lazio 2002; Yao et al. 2017; Pellegrini et al. 2020). However, the electron abundance is lower at the very center of the spirals. The initial magnetic seed field with $0.04 \mu \mathrm{G}$ has evolved into a configuration following the spiral structure of the gas with a magnitude of about $1-10 \mu \mathrm{G}$. This field strength is expected for a Milky Way model (e.g., Beck 2001). The field direction is mostly toroidal, but has no strong reversals as claimed in the magnetic field of the Milky Way (see Sun et al. 2008; Beck \& Wielebinski 2013, and references therein). Consequently, some of our follow-up synthetic synchrotron and RM observations may lack features such as additional zero transition in the RM signal characteristic of the Milky Way. The velocity is well-behaved with an orbital velocity of $220 \mathrm{~km} \mathrm{~s}^{-1}$ within the disk. This is consistent with the velocity 
field of the Galactic disk at a distance of roughly $8 \mathrm{kpc}$ (Bovy et al. 2012) where our Solar System and the observers P1-P4 are located. More recently, Reid et al. (2019) estimated a velocity of $236 \pm 7 \mathrm{kms}^{-1}$. However, a somewhat lower orbital velocity does not substantially affect our synthetic spectral line observations (see Sect. 4.1).

\section{Radiative transfer post-processing}

In this section we briefly outline the post-processing techniques and the underlying physics of the synthetic observations produced by different tracers. The RT simulations are performed with the RT code POLARIS ${ }^{2}$ (Reissl et al. 2016). The advantage of POLARIS is that it unites the physics of dust emission (Reissl et al. 2016, 2018), line transfer including the Zeeman effect (Brauer et al. 2017a,b), and synchrotron emission (Reissl et al. 2019) under a common framework. Furthermore, the code allows one to perform RT simulations on a Voronoi grid. Hence, any AREPO MHD data can be post-processed directly. POLARIS RT simulations consider the polarization state of radiation by default. However, within the scope of this paper we focus only on the unpolarized emission of the different tracers (i.e., dust, synchrotron, and molecular line emission) as well as the Faraday rotation measure $(\mathrm{RM})$.

\subsection{Dust emission}

For the dust component, we assume the canonical model of the ISM. For this model, the ratio of dust mass to gas mass is taken to be $1 \%$. The dust consists of a mixture of materials of $37.5 \%$ silicate and $62.5 \%$ graphite reproducing the characteristics of the Galactic extinction curve (Weingartner \& Draine 2001). The grain sizes $a$ range from $a_{\min }=5 \mathrm{~nm}$ to $a_{\max }=250 \mathrm{~nm}$ with a size distribution following a power law of $N_{\text {dust }}(a) \propto a^{-3.5}$ (Dohnanyi 1969; Mathis et al. 1977). Since we do not intend to track dust polarization, the grains are considered to be spherical for simplicity. We apply absorption cross sections $C_{\text {abs }}(a)$ per grain size pre-calculated on the basis of laboratory data (see Weingartner \& Draine 2001; Reissl et al. 2017, for a detailed description). Assuming equilibrium between absorbed and emitted radiation (Lucy 1999; Bjorkman \& Wood 2001) for the dust emissivity we obtain

$j_{\text {dust }}=B_{v}\left(T_{\text {dust }}\right) \int_{a_{\min }}^{a_{\max }} C_{\text {abs }}(a) N_{\text {dust }}(a) \mathrm{d} a$,

where $B_{v}\left(T_{\text {dust }}\right)$ is the Planck function and $T_{\text {dust }}$ is the dust temperature. The temperature $T_{\text {dust }}$ is calculated on-the-fly in the MHD simulation according to the prescription given in Glover \& Clark (2012), in which heating by gas-grain energy transfer and the absorption of photons from the ISRF is balanced by dust grain thermal emission. For the purposes of calculating $j_{\text {dust }}$ in POLARIS, we simply adopt the values of $T_{\text {dust }}$ computed in the MHD simulation.

\subsection{Synchrotron radiation and Faraday $R M$}

Synchrotron radiation is emitted from relativistic cosmic-ray (CR) electrons following a power-law distribution in energy $N_{\mathrm{el}}(\gamma)=n_{\mathrm{CR}} \gamma^{-3}$, where $\gamma$ is the Lorentz factor (Rybicki \& Lightman 1979; Bennett et al. 2003; Miville-Deschênes et al. 2008). The energy spectrum of the Milky Way is usually taken to be between $\gamma_{\min }=4$ and $\gamma_{\max }=400$ (Webber 1998).

\footnotetext{
2 http://www1.astrophysik. uni-kiel.de/ polaris/
}

The CR electrons of the Milky Way follow a smooth distribution. A parameterization of the $\mathrm{CR}$ distribution is provided in Drimmel \& Spergel (2001). However, the scale height and extension of the disk of the MHD simulations is not the same as that of the Milky Way. Hence, we follow the approach discussed in Reissl et al. (2019) and assume equipartition between the CR energy and the magnetic field energy, so that $n_{\mathrm{CR}} \simeq B^{2} /\left(16 \pi \gamma_{\min } m_{\mathrm{e}} c^{2}\right)$. Here $B$ is the magnetic field in each cell of the model galaxy, $m_{\mathrm{e}}$ is the electron mass, and $c$ is the speed of light. Finally, the synchrotron emissivity is

$j_{\text {syn }}=\int_{\gamma_{\min }}^{\gamma_{\max }} P_{v}(\gamma) N_{\mathrm{el}}(\gamma) \mathrm{d} \gamma$,

where $P_{v}(\gamma)$ is the synchrotron power per frequency $v$ (see, e.g., Pandya et al. 2016; Reissl et al. 2019, for further details). We note that the synchrotron power has an angular dependency $P_{v}(\gamma) \propto$ $\sin \vartheta$, where the angle $\vartheta$ is defined to be between magnetic field direction and the line of sight (LOS).

In the case of polarized radiation, the polarization angle $\chi_{0}$ of that radiation rotates while passing through an ionized and magnetized medium (Rybicki \& Lightman 1979). Consequently, the observed orientation angle becomes $\chi_{\mathrm{obs}}=\chi_{0}+\lambda^{2} \mathrm{ROM}$ (see, e.g., Burn 1966). The quantity

$\mathrm{RM}=\frac{1}{2 \pi} \frac{e^{3}}{m_{\mathrm{e}}^{2} c^{4}} \int n_{\mathrm{el}} B_{\|} \mathrm{d} \ell$

is the wavelength independent rotation measure, $e$ is the electron charge, $n_{\mathrm{el}}$ is the density of free electrons, and $B_{\|}=B \cos \vartheta$ is the magnitude of the magnetic field component along the LOS (Burn 1966; Rybicki \& Lightman 1979; Huang \& Shcherbakov 2011). For the RM calculations we utilize the distribution of $n_{\mathrm{el}}$ provided by the MHD simulation. We define the RM to be positive for LOSs that are parallel with the direction of the magnetic field and negative when anti-parallel. Thus, the RM provides an estimate of the magnetic field strength and the field direction modulated by the distribution of thermal electrons.

\subsection{Molecular line emission and excitation}

We consider the ${ }^{12} \mathrm{CO}$ density distribution $n_{\mathrm{CO}}$ of the MHD galaxy for the line RT. In rapidly rotating disk galaxies, the difference in velocity between molecular clouds located at different points in the disk is generally larger than for the thermal or microturbulent velocities, and so it is generally sufficient to consider only local re-absorption of the emitted photons. This therefore allows us to compute the $\mathrm{CO}$ fractional level populations $f_{\mathrm{i}}$ using the large velocity gradient method (for details, see Ossenkopf 1997; Ober et al. 2015; Brauer et al. 2017b) rather than a full non-LTE treatment. The total emissivity for the transition from level $i$ to $j$ is given by

$j_{\mathrm{ij}}=\frac{h v_{\mathrm{ij}}}{4 \pi} \int_{v_{\min }}^{v_{\max }} n_{\mathrm{CO}} f_{\mathrm{i}} A_{\mathrm{ij}} \phi(v) \mathrm{d} v$,

where the Einstein coefficient $A_{\mathrm{ij}}$ provides the probability of spontaneous emission. For the line broadening we take a Gaussian profile:

$\phi(v)=\frac{c}{\sqrt{\pi} a_{\mathrm{tot}} v_{\mathrm{ij}}} \exp \left(-\frac{c^{2}\left(v-v_{\mathrm{ij}}\right)^{2}}{a_{\mathrm{tot}}^{2} v_{\mathrm{ij}}^{2}}\right)$.

Here $v_{\mathrm{ij}}$ is the characteristic transition frequency and $a_{\mathrm{tot}}^{2}=a_{\mathrm{th}}^{2}+$ $a_{\mathrm{mtrb}}^{2}$ is the line width parameter, where $a_{\mathrm{th}}=\left(2 k_{\mathrm{B}} T_{\mathrm{g}} / m_{\mathrm{mol}}\right)^{1 / 2}$ 


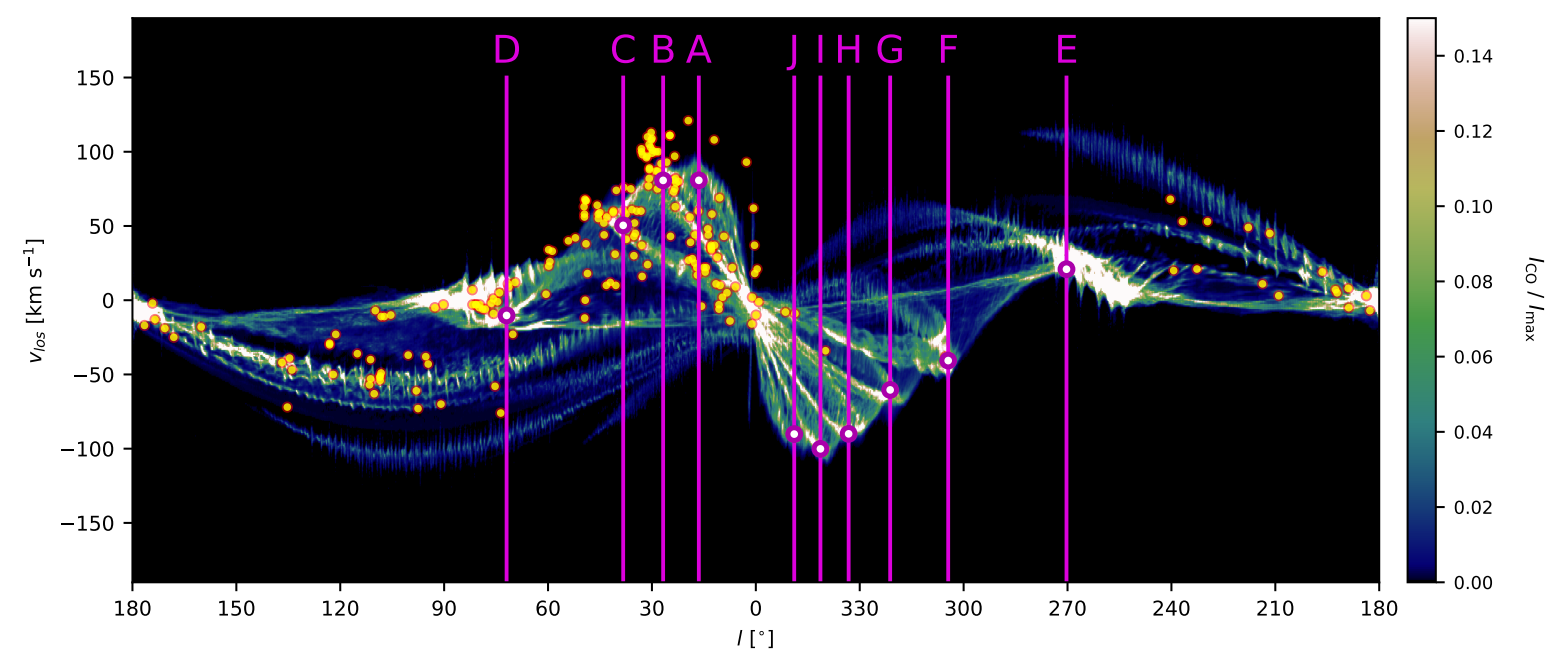

Fig. 3. Synthetic longitude-velocity diagram of the ${ }^{12} \mathrm{CO}(1-0)$ emission line constructed using our Galactic model by placing the observer at position P1 (see Fig. 2). The velocities are averaged within a latitude of $|b|<1.2^{\circ}$. Purple dots and vertical lines show detections of spiral arm tangent points, as determined from CO. Yellow dots represent observations of maser emissions coming from high-mass star-forming regions within our own Milky Way presented in Reid et al. (2019). In general, the detections depend on the tracers used (see Table 1). The detections are labeled A - J with increasing Galactic longitude. The magnitude of the $\mathrm{CO}$ intensity is normalized by its peak value.

accounts for thermal broadening and $a_{\mathrm{mtrb}}$ for microturbulent velocities. We estimate the additional broadening of the lines by microturbulence by following the scaling relation $a_{\mathrm{mtrb}} \approx 0.52 \mathrm{~km} \mathrm{~s}^{-1}\left(L / \mathrm{pc} \times \Sigma / 100 M_{\odot} / \mathrm{pc}^{2}\right)^{1 / 2}$ suggested in Heyer et al. (2009). Here, $L=2(3 V / 4 \pi)^{1 / 3}$ is the characteristic length of a Voronoi cell with volume $V$, and for the surface density $\Sigma$ we take the face-on view of the Milky Way model (see also Fig. 6). For the range of parameters provided by our model we get values of $a_{\mathrm{mtrb}}=1.4 \times 10^{-3}-4.5 \mathrm{~km} \mathrm{~s}^{-1}$ within the disk. Indeed, such a range of the microturbulent broadening parameter $a_{\mathrm{mtrb}}$ is comparable to such values that are commonly assumed to be present in the Milky Way (e.g., Smith et al. 2002; Shetty et al. 2011) justifying the scaling relation approach. We note that the case $a_{\mathrm{mtrb}}>1.0 \mathrm{~km} \mathrm{~s}^{-1}$ only occurs within the densest regions of the spiral arms and the Galactic center. Hence, we do not expect $a_{\text {mtrb }}$ to have a significant impact on our results as the total line width of the $\mathrm{CO}$ emission from an individual molecular cloud in our simulations is dominated by the effects of resolved cloud-scale motions.

For the POLARIS RT simulations we focus on the $J=1 \longrightarrow 0$ transition of ${ }^{12} \mathrm{CO}$ since this molecule is commonly used to trace the ISM mass content, and the Galactic velocity field as well (e.g., Dame et al. 1987, 2001; Jackson et al. 2006; Sormani et al. 2018). The characteristic parameters (i.e., the mass of a CO molecule is $m_{\mathrm{mol}}=4.65 \times 10^{-23} \mathrm{~g}$, the Einstein coefficient $A_{\mathrm{ij}}=7.20 \times 10^{-8} \mathrm{~s}^{-1}$, and the transition frequency $v_{\mathrm{ij}}=115.27 \mathrm{GHz}$ ) are taken from the $\mathrm{LAMDA}^{3}$ molecular database (Schöier et al. 2005), which is also our source for the collisonal excitation and de-excitation rates (Flower 2001; Schöier et al. 2005; Jankowski \& Szalewicz 2005; Wernli et al. 2006). We assume that it is only necessary to account for $\mathrm{CO}-\mathrm{H}_{2}$ collisions since almost all of the $\mathrm{CO}$ in our MHD simulation is located in gas with a high $\mathrm{H}_{2}$ fraction.

\subsection{The ray-tracing scheme}

We simulate all-sky maps for the individual tracers applying the HEALPIX $^{4}$ pixelation scheme (Górski et al. 2005). The number

\footnotetext{
3 https://home.strw.leidenuniv.nl/ moldata/CO.html

4 https://healpix.jpl.nasa.gov/
}

of HEALPIX sides is taken to be $N_{\text {side }}=256$. This results in a total number of 786432 pixels on a sphere larger than the MHD grid, corresponding to an angular resolution of 13.7 arcmin. Each pixel on the HEALPIX sphere defines a LOS between the pixel itself and the distinct pre-defined observer positions labeled P1, P2, P3, and P4. For each of the LOSs we perform ray-tracing by solving the RT problem as outlined above for each individual tracer. Here POLARIS utilizes a Runge-Kutta-Fehlberg solver with built-in error correction working within an error limit of $<10^{-6}$ for each Voronoi cell (see, e.g., Ober et al. 2015; Reissl et al. 2019, for details).

\section{Detection of the Galactic spiral structure}

In this section we present our synthetic observations and evaluate the tangent points as detected using different tracers. These tangent points manifest themselves along the Galactic longitude axis as characteristic peaks, and are usually defined to represent the point where the LOS has exactly one intersection with the spine of a spiral arm. We discuss our results in the context of actual tangent points observed in the Milky Way. For the time being we focus on the observer position P1 representing the most similar conditions to our own environment within the Milky Way. Statistics for the positions P2-P4 are provided later in Sect. 6.

\subsection{CO longitude-velocity diagram}

In Fig. 3 we show the longitude-velocity diagram derived from the synthetic $\mathrm{CO}$ all-sky observations as seen from the observer position $\mathrm{P} 1$ within the latitudinal range of $|b|<1.2^{\circ}$. In detail, we select all pixels within that particular range of $|b|$ of the HEALPIX sphere along the entire longitude $l$ per velocity channel $v_{\text {los }}$ and count the values within a certain bin with $\left(l, v_{\text {los }}\right)$ as coordinates. Furthermore, a latitudinal range of $|b|<1.2^{\circ}$ is chosen to be comparable to the range of latitudes observed in the THOR survey (see Beuther et al. 2016; Wang et al. 2020). We assume the observer to be co-moving with the Galactic disk rotation with a local velocity of $v_{\mathrm{g}}=220 \mathrm{~km} \mathrm{~s}^{-1}$ at $\mathrm{P} 1$. The resulting features are very similar to CO observations of the Milky Way (Dame et al. 1987, 2001) and to the HI emission map shown in 

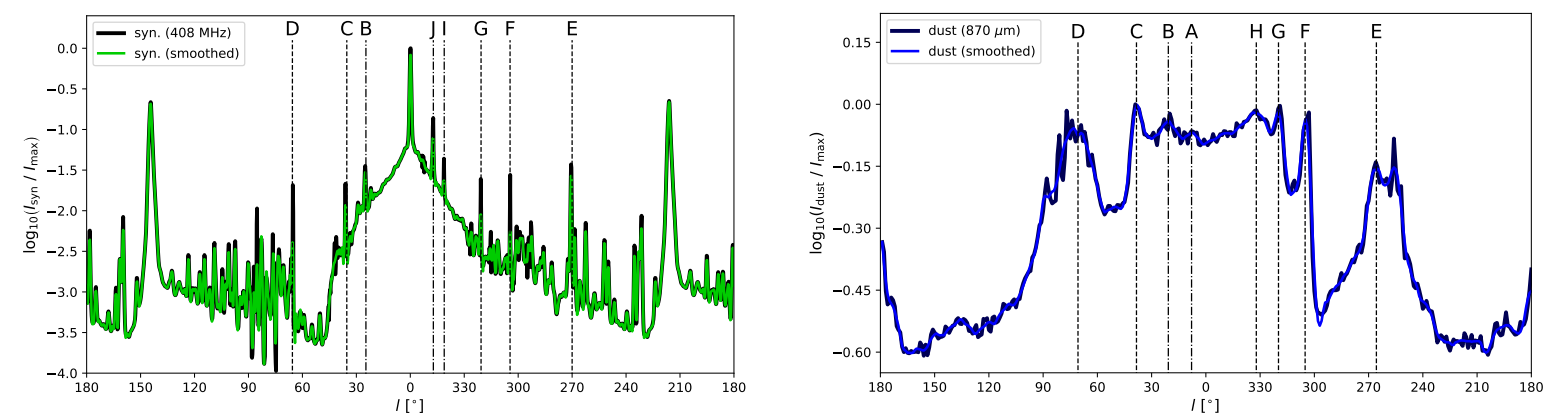

Fig. 4. Longitudinal profiles of the $408 \mathrm{MHz}$ synchrotron emission (left) and $870 \mu \mathrm{m}$ dust emission (right) for our Milky Way model. The panels show the synchrotron emission averaged profile and the dust (blue). The profiles in green and blue are smoothed over $3^{\circ}$ along the longitude. Vertical black dashed lines and letters indicate tangent points of the spiral arms as detected from each tracer. The longitude of the tangent points are not identical in the left and right panels since the exact position depends on the tracer (see Table 1). All profiles are normalized by their peak values for better comparison and analysis.

Reid et al. (2019). For a better comparison between our Galactic model and the Milky Way we also plotted the maser measurements in Fig. 3, as listed in Reid et al. (2019). This comparison reveals that our model underestimates the size of the velocity features toward the Galactic center. However, our Milky Way model does not include the Galactic bar, which is responsible for the high-velocity peaks in the longitude-velocity plots of the central regions (e.g., Sormani et al. 2015a). Furthermore, our overall orbital velocity is somewhat lower than the value suggested in Reid et al. (2019).

The diagram exhibits ten well-defined loops that are the projection of the spiral arms in the longitude-velocity plane. Tangent points are identified by the reversals of the loops (see, e.g., Dame et al. 2001; Sormani et al. 2015a; Reid et al. 2019). The tangent point candidates detected according to this criterion are labeled A-J in order of increasing latitude to facilitate further discussion.

\subsection{Radio observations}

A model of the Milky Way was presented in Beuermann et al. (1985) based on the $408 \mathrm{MHz}$ all-sky radio observation of Haslam et al. (1981, 1982). This model provides an estimate for the Milky Way's spiral arms. We create a similar all-sky map of the $408 \mathrm{MHz}$ synchrotron radiation with the help of POLARIS, which delivers synchrotron emission and RM simultaneously in a single RT simulation (see Reissl et al. 2019, for details). Then we average the synchrotron signal within latitude $|b|<0.2^{\circ}$ and plot it as a function of Galactic longitude. Here, we decrease the latitudinal range compared to $\mathrm{CO}$ in order for the spiral arm to become distinguishable from the synchrotron signal. For a wider range of $|b|$ the signal becomes too noisy for any detection. Furthermore, we smooth the resulting profile along $l$ to get a well-defined maximum for each tangent point detection.

We report that the peaks in our model are much narrower than the peaks presented in Beuermann et al. (1985). This is most likely due to our equipartition assumption implying that the $\mathrm{CR}$ electron distribution $n_{\mathrm{CR}}$ only depends on $B^{2}$ and so CR electrons are mostly present close to the spines of the spiral arms. Hence, we have a scale height that is somewhat smaller than that of the Milky Way and a synchrotron signal may not be detectable from noise for a wider range of $|b|$. A more sophisticated CR propagation scheme may also provide broader peaks comparable to those of Beuermann et al. (1985). Nevertheless, the location of the tangent points can be estimated from the well-defined peaks in the synchrotron signal that are within the orbital cycle of P1 and above the local noise level. The resulting profiles are shown in Fig. 4.
Here, we find that the tangent points $\mathrm{A}$ and $\mathrm{H}$ are not detectable in the synchrotron signal. We speculate that this may be due to the $\sin \vartheta$ dependency of the synchrotron signal. The LOS and the magnetic field directions are not strictly parallel for most of the tangent points, i.e., the angular dependency of the synchrotron emission is $\sin \vartheta>0$. Hence, the strong increase in the magnetic field strength can compensate for the decreasing factor coming from the angular dependency and most tangent arms can be detected by synchrotron observations. However, this is not the case for the tangent point $\mathrm{H}$ where $\sin \vartheta \approx 0$.

\subsection{Cold dust signatures}

Tangent points may also be detected in emission from cold dust. Corresponding dust observations of 240 and $870 \mu \mathrm{m}$ emission are provided in Drimmel (2000) and Beuther et al. (2012), respectively. However, Beuther et al. (2012) counted the dust emission of distinct sources, whereas we take the profile of the continuum emission along the entire galactic plane. The procedure of detecting the tangent points along the longitude is similar to that of the synchrotron detections. Again, we consider the range of $|b|<1.2^{\circ}$ as in the THOR survey (Beuther et al. 2016; Wang et al. 2020).

In Fig. 4 we present the profile of the $870 \mu \mathrm{m}$ emission. In contrast to Beuther et al. (2012), we do not find a central peak. This may be because our MHD simulations lack a proper bar and bulge model. However, the profile has some well-defined local maxima that can be clearly associated with the tangent points of the spiral arm candidates A-H. The synthetic profile of the $240 \mu \mathrm{m}$ dust emission is virtually identical to the $870 \mu \mathrm{m}$ profile for our Milky Way model. Hence, we discuss only the $870 \mu \mathrm{m}$ dust observations in the following sections.

\subsection{Longitudinal Faraday RM profiles}

Faraday rotation is sensitive to the magnetic field strength $B$, electron fraction, and the direction of the field lines with respect to the LOS (i.e., $\cos \vartheta$ ). Within the spiral arms the field strength $B$ reaches a maximum and $\cos \vartheta \approx \pm 1$ (see Fig. 2). Consequently, we expect the RM profile to have well-defined features corresponding to the tangent points coming mostly from the magnetic field properties. However, this may only be true for our model and might not match the situation in the Milky Way arms themselves, where an enhanced electron density may also contribute to the RM signal (Shanahan et al. 2019).

Oppermann et al. (2012) produced an all-sky map of Galactic Faraday rotation using all available RM data. This all-sky 

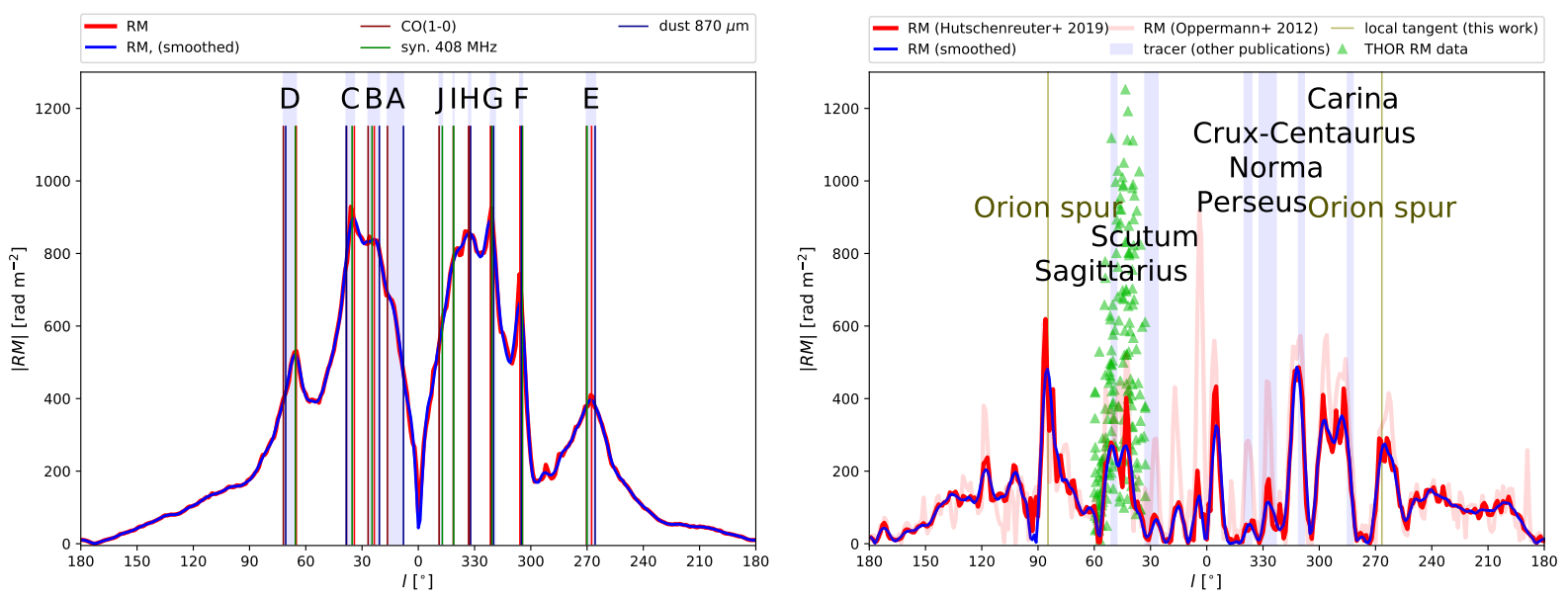

Fig. 5. Left: synthetic RM profile of our Milky Way model as a function of longitude $l$. Vertical lines represent the tangent point detections as derived from the distinct tracers listed in Table 1. Vertical shaded bars and labels indicate the angular range of the detections for all tracers per spiral arm. Right: same as the left panel, but for the observed Milky Way RM (Oppermann et al. 2012; Hutschenreuter \& Enßlin 2020). Green triangles indicate the THOR RM data presented in Shanahan et al. (2019) as well as newly derived data points. Some of the THOR data points beyond $1300 \mathrm{rad} \mathrm{m}^{-2}$ were cut for better comparability with the left panel.

map was later improved by taking the free-free emission into account (Hutschenreuter \& Enßlin 2020). Conveniently, the RM of Oppermann et al. (2012) and Hutschenreuter \& Enßlin (2020) are publicly available in the HEALPIX format ${ }^{5}$. As for the other tracers we create longitudinal profiles by averaging all pixels within the range of $|b|<1.2^{\circ}$.

In Fig. 5 we compare synthetic RM profiles (left panel) with Galactic RMs derived by Oppermann et al. (2012) and Hutschenreuter \& Enßlin (2020) (right panel). The RM profiles in Fig. 5 are shown in comparison with the set of tangents derived from the other tracers and the observed Milky Way arm tangent points as presented in Beuermann et al. (1985), Taylor \& Cordes (1993), Drimmel (2000), Beuther et al. (2016), Hou \& Han $(2014,2015)$, and references therein.

In contrast to the observed RM profile from Hutschenreuter \& Enßlin (2020) (right panel), our synthetic map (left panel) displays an increase in the magnitude of the Faraday RM toward the center. This is a result of the strong increase in the magnetic field strength toward the center of our Milky Way model galaxy since we are not properly modeling the bulge region. We also note that our magnetic field is mostly toroidal, and lacks reversals of the field lines within the plane of the disk as predicted by other Galactic models (e.g., Jansson \& Farrar 2012; Grand et al. 2017). Hence, we are missing some of the structure coming from such reversals in our RM profile. The minimum of the profile at $1=0^{\circ}$ indicates the change in sign of the RM where the magnetic field direction goes from parallel $(\cos \vartheta>0)$ to anti-parallel $(\cos \vartheta<0)$ with respect to the LOS (see Eq. (3) and Fig. 2). The synthetic RM profile in Fig. 5 has well-defined maxima. Assuming that these maxima correspond to the spiral arm tangents, we detect the candidates B-H.

The Galactic RMs of Oppermann et al. (2012) and Hutschenreuter \& Enßlin (2020) also show peaks in the Galactic plane along the directions of known spiral arms. The width of these peaks in longitude varies significantly, and it does not appear to be correlated with the longitude extent of spiral arm

\footnotetext{
5 Oppermann map:

wwwmpa.mpa-garching.mpg.de/ift/faraday/

Improved Hutschenreuter map:

wwwmpa.mpa-garching.mpg.de/ ensslin/research/data/

faraday_revisited.html
}

tracers for each arm. The height of the peaks varies from arm to arm, while some RM peaks of similar magnitude appear not related to any known arm. A detailed discussion of each spiral arm tangent is beyond the scope of this paper. However, we make two comments that are significant when comparing the left and right panels of Fig. 5.

First, the magnitude of the fluctuations in the RMs of Oppermann et al. (2012) and Hutschenreuter \& Enßlin (2020) is of the same order of magnitude as RMs from individual HII regions (see, e.g., Shanahan et al. 2019, for a discussion). It is therefore possible that some observed RM peaks are related to individual HII regions along the LOS. These are not modeled in the present simulation.

Second, Shanahan et al. (2019) showed that some of the surveys included by Oppermann et al. (2012) and Hutschenreuter \& Enßlin (2020) were biased against very high $|R M|$. This bias was introduced by the use of data from the NRAO VLA Sky Survey (NVSS), which is limited to $|R M| \lesssim 400 \mathrm{rad} \mathrm{m}^{-2}$ (in the case of Taylor et al. 2009) or by selection of polarized sources from the NVSS source catalog for new observations. The recent survey by Schnitzeler et al. (2019) explicitly searched for targets with $|R M|<1000 \mathrm{rad} \mathrm{m}^{-2}$. The Bayesian analysis cannot correct a bias in the data, if it exists, as in the case of the Sagittarius arm.

\section{Analysis of arm properties}

In this section we discuss the characteristic profile shapes associated with tangent points and a method for recovering the pitch angles of the spiral arms on the basis of the tangent points.

\subsection{The Milky Way from an outside perspective}

In addition to the all-sky maps, we create a face-on map of the column density $N_{\mathrm{H}}$ as seen from an outside observer. In Fig. 6 we show the column density map and depict the tangent points detected from the perspective of observer P1. For reference, in this section we additionally consider the tangent points as determined from the maxima in the longitudinal profile of $N_{\mathrm{H}}$. All tangent points of the different tracers are listed in Table 1 for the observer P1 (the statistic of the other observer positions is discussed in Sect. 6). Comparing the tangent points detected by 
Table 1. Tangent points of spiral arms in our Milky Way model as determined using different tracers as seen from the example observer position P1.

\begin{tabular}{|c|c|c|c|c|c|c|c|c|c|}
\hline \multicolumn{2}{|c|}{ Tangent point $\mathrm{CO}(1-0)$} & \multicolumn{2}{|c|}{$\mathrm{N}_{\mathrm{H}}$} & \multicolumn{2}{|c|}{$\mathrm{RM}$} & \multicolumn{2}{|c|}{ Syn. $408 \mathrm{MHz}$} & \multicolumn{2}{|c|}{ Dust $870 \mu \mathrm{m}$} \\
\hline & $1\left[^{\circ}\right]$ & $1\left[^{\circ}\right]$ & $\Delta \mathrm{l}\left[\left[^{\circ}\right]\right.$ & $1\left[^{\circ}\right]$ & $\Delta 1\left[^{\circ}\right]$ & $1\left[^{\circ}\right]$ & $\Delta l\left[\left[^{\circ}\right]\right.$ & $1\left[^{\circ}\right]$ & $\Delta 1\left[^{\circ}\right]$ \\
\hline A & 16.46 & $\mathrm{X}$ & $\mathrm{X}$ & $\mathrm{X}$ & $\mathrm{X}$ & $\mathrm{X}$ & X & 7.91 & 8.55 \\
\hline B & 26.76 & $\mathrm{X}$ & $\mathrm{X}$ & 23.47 & 3.29 & 24.68 & +2.08 & 20.76 & +6.00 \\
\hline $\mathrm{C}$ & 38.30 & 38.38 & -0.08 & 34.11 & +4.19 & 35.31 & +2.99 & 38.38 & -0.08 \\
\hline $\mathrm{D}$ & 71.97 & 74.45 & -2.48 & 65.08 & +6.89 & 65.56 & +6.41 & 70.72 & +1.25 \\
\hline $\mathrm{E}$ & 270.30 & 264.01 & +6.29 & 267.88 & +2.42 & 270.00 & +0.30 & 265.69 & +4.61 \\
\hline $\mathrm{F}$ & 304.46 & 304.30 & +0.16 & 305.81 & -1.35 & 304.52 & -0.06 & 305.11 & -0.65 \\
\hline $\mathrm{G}$ & 321.21 & 319.48 & +1.73 & 321.53 & -0.32 & 320.65 & +0.56 & 319.83 & +1.38 \\
\hline $\mathrm{H}$ & 333.20 & 329.75 & +3.45 & 332.62 & +0.58 & X & X & 332.11 & +1.09 \\
\hline I & 341.35 & X & X & X & X & 341.13 & 0.22 & X & X \\
\hline $\mathbf{J}$ & 348.91 & $X$ & $X$ & $X$ & $X$ & 347.18 & 1.73 & $X$ & $X$ \\
\hline
\end{tabular}

Notes. The longitude of the tangent point is indicated by 1 , while $\Delta \mathrm{l}$ gives the difference with respect to the values determined from $\mathrm{CO}(1 \rightarrow 0)$ (see Fig. 3). The X flags tangents with no detection. We note that the tabulated angles merely represent our Milky Way analog and are not to be directly compared with actual observations of the Milky Way.

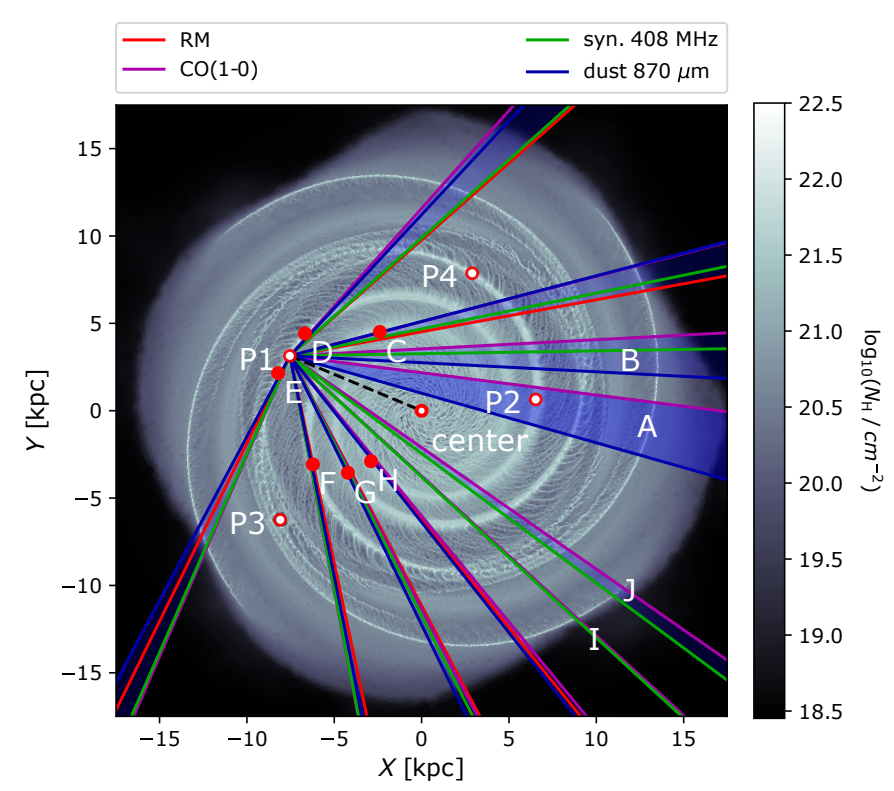

Fig. 6. Face-on column density map of our Milky Way model. Red circles are the observer positions and the Galactic center, while red dots represent the tangent points. The black dashed line represents the LOS toward the center (i.e., $1=0^{\circ}$ in the Galactic coordinate system). Colored lines indicate the detected spiral arms for different tracers, and the blue shaded areas show the angular range of detections for each spiral arm.

different tracers, we find a maximum scatter of about $\pm 6^{\circ}$, which is comparable to the range presented in $\operatorname{Hou} \& \operatorname{Han}(2014,2015)$, among others, for the Milky Way. Comparing Fig. 6 with Figs. 4 and 5, it is apparent that the spiral arm tangents closer to P1 (D and $\mathrm{E}$ ) are broader in longitude, while the most distant spiral arm tangents in the inner Galaxy (A and B, or H, I, and G) appear to blend together. The perspective of the observer is important in the sense that spiral arm tangents near longitudes $\pm 45^{\circ}$ display the highest contrast.

Comparing the tangent points as detected from the embedded perspective of observer P1 (defined as peaks in longitudinal profiles of various tracers) with the face-on map reveals that only the points $\mathrm{C}, \mathrm{D}, \mathrm{E}, \mathrm{F}, \mathrm{G}$, and $\mathrm{H}$ are actually associated with clearly defined spiral arms. The tangents A, B, I, and J are associated with blurred arms that blend into the disk of diffuse material near the Galactic center in the MHD simulation. We recall that in our Milky Way model we are missing a proper bar and bulge as provided in the numerical simulations of Sormani et al. (2015b), among others. However, such simulations do not include a magnetic field component.

The arm structure of the Milky Way outside the central bar region can be approximately described by logarithmic spirals (Vallée 2015; Hou \& Han 2015). This is also the model we adopt in this study, whereas the log-periodic spirals suggested in Reid et al. (2014) would also account for a possible kink within a spiral arm. Following trigonometrical considerations the pitch angle $\Psi$ of any arm may be estimated by

$\Psi=\tan ^{-1}\left[\frac{\ln \left(\sin \left(l_{180}\right) / \sin \left(2 \pi-l_{360}\right)\right)}{\left(l_{180}-l_{360}+\pi\right)}\right]$.

Here, the quantity $l_{180}$ is the longitude of a tangent point between $0^{\circ}$ and $180^{\circ}$ and $l_{360}$ is a tangent between $180^{\circ}$ and $360^{\circ}$. Consequently, the two tangent points $l_{180}$ and $l_{360}$ are required to belong to the same spiral arm, which runs from one tangent point to the other. For example, the tangent points $\mathrm{C}$ and $\mathrm{F}$ belong to the same arm (hereafter called $\mathrm{ARM}_{\mathrm{CF}}$ ) and $\mathrm{D}$ and $\mathrm{E}$ constitute the tangent points of the arm $\mathrm{ARM}_{\mathrm{DE}}$. We note that $\mathrm{ARM}_{\mathrm{DE}}$ is the local arm of the observer position $\mathrm{P} 1$. The arms corresponding to the tangent points $\mathrm{G}$ and $\mathrm{H}$ possess no second tangent point, similarly to the Perseus and Norma arms in our own Galaxy (see Fig. 1).

The pitch angles $\Psi$ corresponding to the various spiral arms in our Milky Way model are listed in Table 2. All these values are determined using Eq. (6) with the exception of $N_{\mathrm{H}}$, which is determined from the face-on map. The arm $\mathrm{ARM}_{\mathrm{CF}}$ has an average pitch angle of $\Psi=11.72^{\circ}$ and the local arm $\mathrm{ARM}_{\mathrm{DE}}$ has $\Psi=11.73^{\circ}$. These are average values calculated from various tracers but without the contribution of the $N_{\mathrm{H}}$ data, which has to be excluded since these angles are not derived from the perspective of an inside observer. The recovered pitch angles of $\mathrm{ARM}_{\mathrm{CF}}$ and $\mathrm{ARM}_{\mathrm{DE}}$ fall well within the range of pitch angles of $7^{\circ} \lesssim \Psi \lesssim 15^{\circ}$ reported for the spiral arms of the Milky Way (e.g., Taylor et al. 2009; Hou \& Han 2014; Yao et al. 2017; Chen et al. 2019; Reid et al. 2019). Similar values of $\Psi$ are found for other spiral galaxies, e.g., $\Psi<25^{\circ}$ for M83 (Frick et al. 2016). 
Table 2. Pitch angle of the spiral arms $A R M_{C F}$ and $A R M_{D E}$ for the different considered tracers as seen from the observer position P1.

\begin{tabular}{ccc}
\hline \hline Tracer & ARM $_{\mathrm{CF}}$ & $\mathrm{ARM}_{\mathrm{DE}}$ \\
\hline$N_{\mathrm{H}}$ & $10.78^{\circ}$ & $10.80^{\circ}$ \\
$\mathrm{CO}(1-0)$ & $10.75^{\circ}$ & $8.94^{\circ}$ \\
$\mathrm{RM}$ & $12.98^{\circ}$ & $13.70^{\circ}$ \\
Syn. $408 \mathrm{MHz}$ & $12.83^{\circ}$ & $12.41^{\circ}$ \\
Dust $870 \mu \mathrm{m}$ & $10.33^{\circ}$ & $11.86^{\circ}$ \\
\hline Mean $\pm \mathrm{STD}$ & $11.72^{\circ} \pm 1.38^{\circ}$ & $11.73^{\circ} \pm 2.01^{\circ}$ \\
\hline
\end{tabular}

Notes. Here the arm $\mathrm{ARM}_{\mathrm{CF}}$ is defined by the tangent points $\mathrm{C}$ and $\mathrm{F}$, whereas $\mathrm{ARM}_{\mathrm{DE}}$ is the local arm of observer P1 with tangent points $\mathrm{D}$ and E. Mean values and the standard deviations (STD) are calculated without the angles of $N_{\mathrm{H}}$.

The $\Psi$ values determined from the perspective of an embedded observer are very close to the values determined from the face-on $N_{\mathrm{H}}$ map. This validates the method provided by Eq. (6) to estimate the pitch angle of spiral arms. However, we note that the systematic offset illustrated by our geometrical model presented in Sect. 5.3 has a significant effect on the recovered value of the pitch angle. For any inner arm border close to the center we find a pitch of $\Psi \approx 12^{\circ}$, whereas for the outer arm border we find values closer to $\Psi \approx 9^{\circ}$. This is not a dramatic error considering that the tracers do indeed probe different regions within a spiral arm. The derived angles $\Psi$ presented in Table 2 fall well within the actual observed rang of $9^{\circ}<\Psi<12^{\circ}$. However, it illustrates the importance of taking into account the systematic geometric effect explained in Fig. 1 and the difference between the various tracers when determining the pitch angle of the Milky Way spiral arms using Eq. (6).

Furthermore, for $\mathrm{ARM}_{\mathrm{CF}}$ and $\mathrm{ARM}_{\mathrm{DE}}$, the pitch angle of the gaseous spiral arms are close to the pitch angle of $\Psi=15^{\circ}$ applied in the gravitational potential of the MHD simulation (see Sect. 2.2). However, they are not identical; the pitch angle of the gas is smaller than the pitch angle $\Psi=15^{\circ}$ of the applied spiral potential (see Sect. 2.1). It was noted in Roberts (1969) and Sormani et al. (2017) that the gas may shock in front of or even behind the minimum of the potential well. A similar explanation for an observed offset between gas content and star formation tracers in the Milky Way is discussed in Ragan et al. (2018) and the references therein in greater detail. The exact position of the shock strongly depends on the local parameters such as the sound speed. In Fig. 2 we see that the plotted spirals indicating the potential are not always congruent with the density maximum of the spiral arms. We speculate that this offset between the potential minimum and the shock may account for the deviation between the pitch angle present in the applied potential and the pitch angles recovered from the observational tracers.

\subsection{The pitch angle of the Milky Way's Local Arm}

In the previous section, we showed that the pitch angle of any local arm may be derived from the synthetic RM profile. We note two particular features in the RM profile of the Milky Way at $84.68^{\circ}$ and $266.61^{\circ}$ (indicated in right panel of Fig. 5 for comparison). These features, are clearly within the solar orbit and we speculate that they originate from our own Local Arm, the Orion spur (see Fig. 1). Consequently, both features should belong to the same spiral arm and we can apply Eq. (6) to determine its pitch angle. This results in a pitch angle of $\Psi=4.36^{\circ}$ for the Orion spur.
This finding is well within the range of pitch angles proposed in the literature by studies utilizing various tracer techniques. In Yao et al. (2017) a pitch angle of $\Psi=2.77^{\circ}$ is derived by modeling the free electrons in the Milky Way; Chen et al. (2019) report a value of $\Psi=10.20^{\circ}$ based on the distribution of observed O/Btype stars; and Reid et al. (2019) estimate an angle of $\Psi=11.40^{\circ}$ by observing molecular masers of young high-mass stars. Hence, the features in the RM signal of the Oppermann et al. (2012) and Hutschenreuter \& Enßlin (2020) maps may indeed trace the Orion spur. We note that our determination of the pitch angle of the Orion spur may be affected by an angular offset as discussed in the following section.

\subsection{Is there a characteristic spiral arm signature?}

The RT simulated RM profile in Fig. 5 shows distinct peaks at spiral arm tangents identified in the simulation. The peaks of tangents $\mathrm{C}, \mathrm{G}$, and $\mathrm{F}$ are distinctly asymmetric, with a much steeper RM gradient on the outside. The RM peaks associated with the local arm (tangents D and E) are broad and symmetric. The RM profiles of the tangent points in the inner galaxy appear blended. In Fig. 7 we zoom in on the peak associated with tangent point $\mathrm{F}$, which is most similar to the Sagittarius arm tangent observed by Shanahan et al. (2019) in terms of viewing angle with respect to the Galactic center (and therefore distance from the Sun and the Galactic center).

The red curve in Fig. 7 shows the synthetic RM as a function of longitude in relation to the THOR RM data of the region. For the benefit of this paper, RMs from the THOR survey that were derived since the work of Shanahan et al. (2019), but following their procedure, are also shown as green dots. An extensive THOR RM catalog will be published in a future paper. Also plotted is the synthetic dust profile (blue) for comparison. The synthetic data sets are mirrored so that they are centered on $l=48.6^{\circ}$.

Coming from the Galactic outskirts $\left(l>48.6^{\circ}\right)$ our RT model reproduces the low RM on the outside of the arm, the peak, and the higher RM for lines of sight that intersect the arm at lower longitude. The peak itself is not as sharp as the THOR data. A similar trend can be seen in the trend for the $870 \mu \mathrm{m}$ dust profile.

The THOR RMs indicate a pattern of enhanced mean RM and enhanced RM variance for lines of sight through the arm $\left(l \lesssim 46^{\circ}\right)$ compared with lines of sight that do not intersect the $\operatorname{arm}\left(1 \gtrsim 50^{\circ}\right)$, as noted by Shanahan et al. (2019). Near the spiral arm tangent, a strong excess in mean RM and RM variance is seen. The increased RM variance may be the result of smallscale structure such as HII regions in the spiral arm. Note that the synthetic RM profile is expected to reproduce the mean RM, not the excesses due to small scale structure. The highest RMs up to $4219 \mathrm{rad} \mathrm{m}^{-2}$ may be affected by the proximity of the large HII region complex W51 as reported by Shanahan et al. (2019). The simulations, as represented by the red curve, trace the gradual increase in RM toward lower longitude well. They also show an excess RM at the arm tangent that is significant compared with the RM of the remainder of the disk (see also Fig. 5 for wider context). The excess Faraday rotation in the simulations is, however, smaller than indicated by the data.

To quantify this, we fitted the parameters to the longitudinal fit function ${ }^{6} f(l)=a+b \times \exp \left[-(c+l)^{2}\right]+d \times l^{5}$ to the complete THOR data set (both Shanahan et al. 2019 data and newly

\footnotetext{
6 The parameters as well as the fit function itself were determined with the help of MATHEMATICA Version 11.0, https://wwW. wolfram. com/mathematica
} 


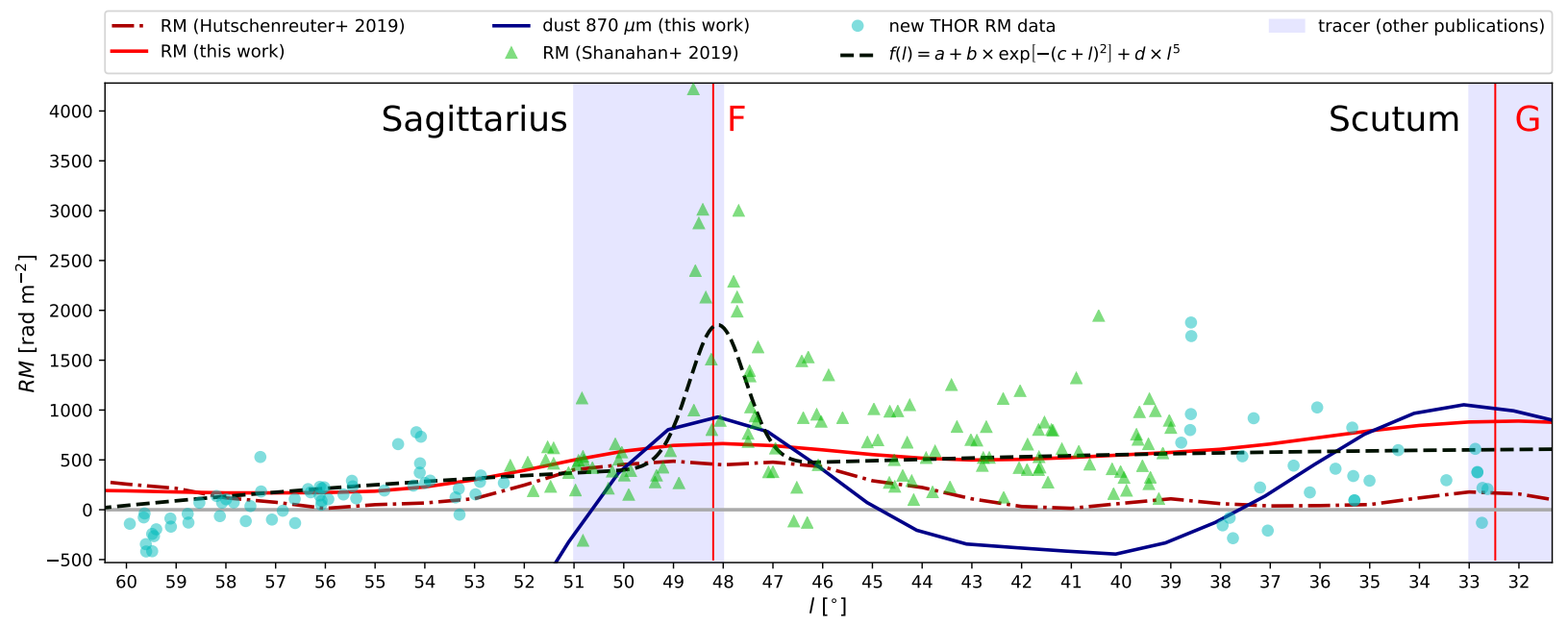

Fig. 7. Synthetic and measured RM values. The synthetic RM (solid red) and synthetic dust (solid blue) are compared to the RM observations (dashdotted brown) derived from the Hutschenreuter \& Enßlin (2020) map and the THOR survey, respectively. The THOR data contains measurements (green triangles) already published in Shanahan et al. (2019) as well as newly calculated RM values (green dots). All synthetic profiles are shifted and mirrored to match their tangent point F, which is the peak value of the Shanahan et al. (2019) data at $l=48.6^{\circ}$ in the Galactic coordinate system. For comparison, the synthetic dust profile shown in Fig. 4 is magnified to match the RM data. The parameters of the function $f(l)$ are fitted to the full THOR data set (dashed black). Vertical red lines indicate the tangent points F and G of the synthetic RM profile shown in Fig. 5, whereas vertical blue bars indicate the range of tangent points of different tracers for the Sagittarius and the Scutum arm of the Milky Way.
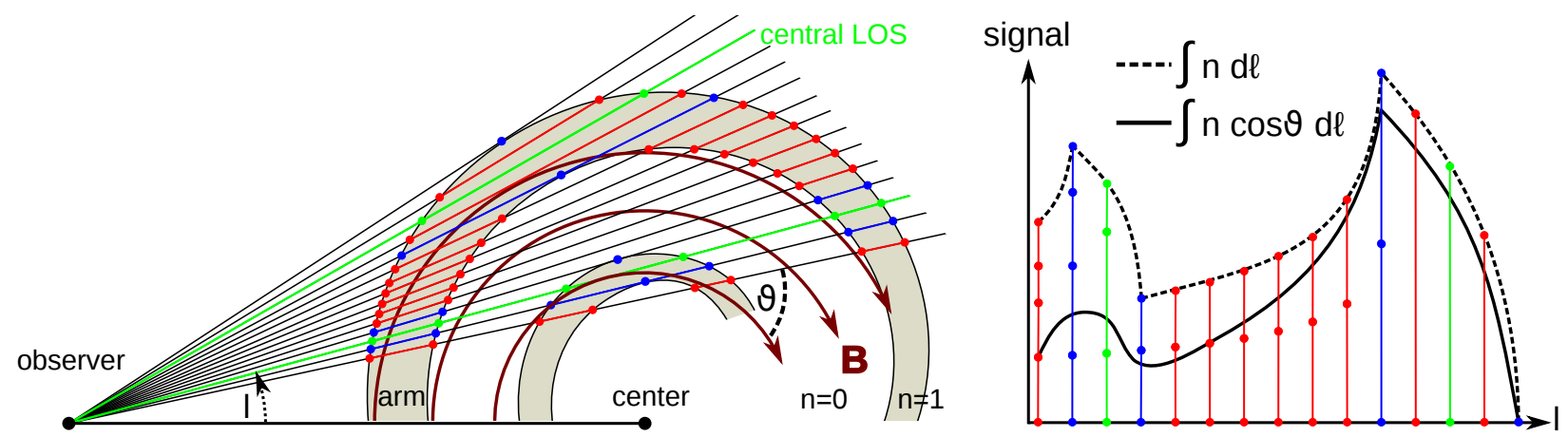

Fig. 8. Schematic sketch of the toy model (left) illustrating the origin of the characteristic shark-fin spiral arm profile (right). Black lines are LOSs in the Galactic plane converging toward the observer, blue lines mark the inner and outer "tangents" of the spiral arm, and the green line is the LOS through the center of the arm. The arms have an arbitrary density of $n=1$ a.u. where as the interarm regions remain empty. The arms follow a logarithmic spiral where as the direction of the magnetic field $\boldsymbol{B}$ is purely toroidal. The distinct LOSs and $\boldsymbol{B}$ define the angle $\vartheta$. Colored dots indicate the intersection of the different LOSs and the arm borders. The diagram on the right shows a re-constructed signal along the longitude 1 . The dashed black line is calculated assuming that the signal strength is proportional to the length of the portion of the LOSs which lies within the spiral arm. The dashed line is weighted by $\cos \vartheta$. Note that the peak in the observed signal corresponds to the inner tangent (blue), and not to the central LOS (green) as usually assumed.

calculated RMs). Indeed, the trend may also be represented by another function, e.g., by a polynomial. The point is, that the fit reveals a comparable trend with that of our simulated RM profile with a steady increase resulting in a maximum and followed by a further increase toward the galactic center. We note that a similar pattern is also present in the dust profile.

We speculate that such a shape is primarily a result of the geometry of the arm itself. In order to test this hypothesis we construct a simple 2D arm model consisting of two identical logarithmic spirals. A schematic illustration of this model is provided in Fig. 8. The spiral arms are assumed to have a finite width. We assume a constant density of unity within the spiral arms while the interarm regions remain empty. Then, we trace rays through the arms toward a the observer position for different equidistant longitudes 1 . Hence, the tracer signal in this toy model directly scales proportionally to the length of the path which lies within the arm.
Plotting this signal as a function of the longitude $l$ we find in Fig. 8 a characteristic shape of a rise and subsequent decline resembling a shark fin. We refer to this as the shark-fin profile. It has a peak and a shoulder in the direction of the Galactic center, similar to the profiles of our Galactic model and the data of Shanahan et al. (2019) as well as the dust peaks in Fig. 4. Naturally, the profile of our simple toy model is not exactly what we see in Figs. 4 and 7 since we ignored secondary effects from the magnetic field, gas density, electron fractions, and dust distribution. Repeating the calculation of the signal weighted by $\cos \theta$ leads to an decrease in magnitude and the second peak corresponding to the inner tangent of the arm becomes smoothed. This peak resembles no longer the shark-fin profile but is more comparable to those seen in Oppermann et al. (2012) and Hutschenreuter \& Enßlin (2020), respectively.

In the case of the RM the angle of the magnetic field with the line of sight modifies the shark fin profile somewhat. The main 

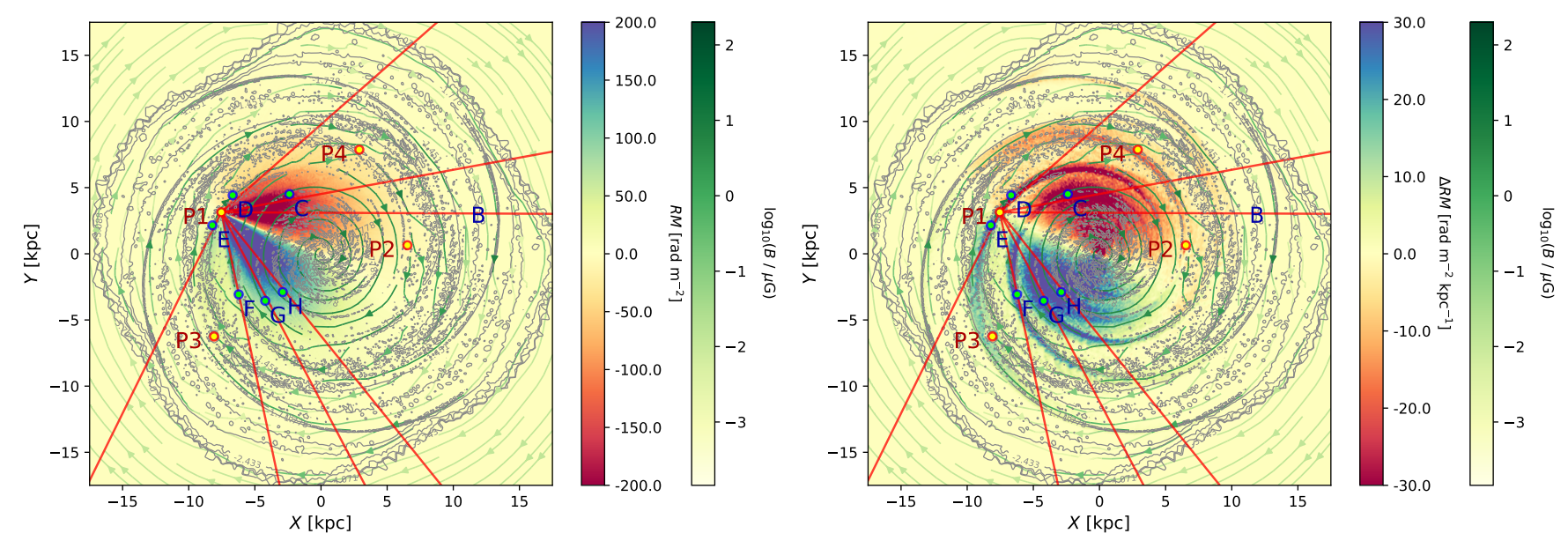

Fig. 9. Left panel: accumulated values of the RM along all LOSs that lie within the Galactic disk plane. All LOSs start outside the MHD grid with $\mathrm{RM}=0 \mathrm{rad} \mathrm{m}^{-2}$ and incrementally converge with each step size $\mathrm{d} \ell$ inward at the very observer position P1 where the RM reaches its maximum. The magnitude of the resulting RM signal at position P1 is shown in Fig. 5, left panel. Gray contours indicate the electron density and the vector field represents the magnetic field strength and orientation (see Fig. 2). Red lines are the tangents B-H detected by the RM signal, whereas blue dots represent the tangent points derived from the column density map shown in Fig. 6. Right panel: same as the left panel, but the relative change $\Delta \mathrm{RM}=(\mathrm{RM}(\ell+\mathrm{d} \ell)-\mathrm{RM}(\ell)) / \mathrm{d} \ell$ is plotted along all LOSs as they approach $\mathrm{P} 1$.

feature to recognize is that for lines of sight that intersect the arm twice, the tangent point where $\vartheta \approx 0$ is absent. The strongest effect is therefore in the wing of the shark fin profile. This is illustrated in Fig. 8 for a magnetic pitch angle of $0^{\circ}$.

An interesting aspect that follows from our 2D toy model is that the peak in the profile is not associated with a line of sight through the center of the arm, but represents the inner border of a spiral arm (see also Hou \& Han 2015). Consequently, all observations of dust, RM, and also synchrotron tracers may systematically underestimate the Galactic longitude of the tangent of the spine of a spiral arm by a few degrees (assuming that the quantity of interest is the center of the spiral arm).

It may even be possible to derive an arm width from the shape of the profile by similar geometric considerations. However, quantifying the exact parameters for an offset angle or an arm width would require an extensive systematic evaluation of RT models and observational data alike, and is therefore beyond the scope of this paper.

\subsection{The origin of the RM signal}

We repeat the RT post-processing for the RM runs. In the previous runs all rays start outside the grid and converge at the very positions of the observers. Now we stop the RT runs at the surface of a sphere surrounding the observer with a distinct pre-defined radius. This way, the signal represents the accumulated contribution of the RM up to this particular sphere. Hence, incrementally increasing the radius of the sphere allows us to investigate the origin of the RM signal within our Milky Way model.

In Fig. 9 we present the accumulated increase in the RM signal toward the observer $\mathrm{P} 1$ as well as the relative change $\Delta \mathrm{RM}$ between adjacent spheres in the $X Y$-midplane of the Galactic disk simulation. The accumulated signal coming from the direction of the Galactic center starts to reach a considerable amount of RM within a distance of roughly $10 \mathrm{kpc}$ from P1. Hence, we note that Fig. 9 has to be taken with care since other RM modeling efforts as presented in Pakmor et al. (2018), among others, indicate that a significant part of the RM signal may even originate at about $15 \mathrm{kpc}$ from the observer. However, in our model the accumulation of RM is mostly due to the increase in the magnetic field strength near the center of our Milky Way model (see also Fig. 2). Consistent with the $\cos \vartheta$-dependency of the Faraday RM (see Eq. (3)) we see no signal at all from the very center of the galaxy.

Faraday RM is significantly enhanced in the spiral arms when compared with interarm regions. This becomes even more obvious in the plot of the relative change $\Delta \mathrm{RM}$ of the signal shown in Fig. 9. The distribution of $\triangle \mathrm{RM}$ within the Galactic disk matches the spiral structure of the Milky Way model. We also note some asymmetry for the observer position P1 along the tangents $\mathrm{D}$ and $\mathrm{E}$, with a smaller increase $\triangle \mathrm{RM}$ in the direction of tangent E. This is also an effect of the magnetic field strength since the strength increases as the arm spirals from the outskirts toward the center. This result is also consistent with the longitudinal profile of RM in Fig. 5 where the peak of the tangent $D$ is larger than that of $\mathrm{E}$. In conclusion, the peaks $\mathrm{C}-\mathrm{H}$ of the longitudinal RM signal are indeed associated with the tangent points within the spiral arms.

The RM signal is linearly dependent on electron density $n_{\mathrm{el}}$ and the LOS magnetic field strength $B_{\|}$. However, we find a Pearson correlation of $r=0.91$ between $|\Delta \mathrm{RM}|$ and $\left|B_{\|}\right|$, but only a $r=0.10$ between $|\Delta \mathrm{RM}|$ and $n_{\mathrm{el}}$ for the disk midplane shown in Fig. 9. Hence, the spiral arm detection by means of RM observations in our Milky Way model is mostly a consequence of the increase in magnetic field strength within the arms. We note that this particular aspect of our MHD model may not accurately represent the Milky Way where the density of free electrons $n_{\mathrm{el}}$ is also found to be higher within the arms (see, e.g., Cordes \& Lazio 2002; Langer et al. 2017). Thus, the RM signal in the Milky Way may be much more influenced by electron fractions than in our synthetic observations suggest.

\section{The impact of a local bubble}

In this section we discuss the influence of the local conditions of the observers on our synthetic observations. The sensitivity of synthetic Galactic synchrotron and line observations to the presence of such a local bubble has also been discussed in Reissl et al. (2019) and Pellegrini et al. (2020). These authors found that 

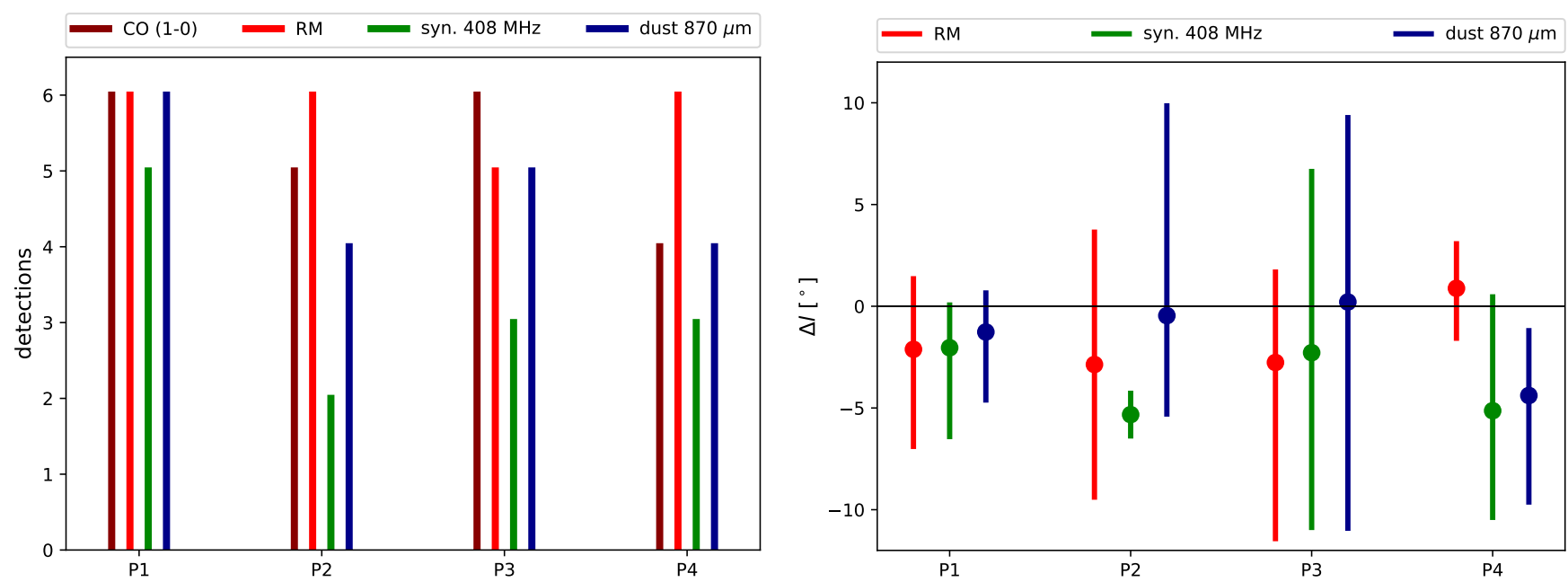

Fig. 10. Left: number of spiral arm detections per tracer for the different observer positions P1, P3, P3, and P4, respectively. Here, only the tangent points $\mathrm{C}-\mathrm{H}$ are considered leading to a maximum of six possible detections. Right: deviation of tangent point angles with respect to the angle of $\mathrm{CO}$ of all spiral arms. Vertical lines represent the minimal and maximal deviation for each of the four observer positions P1-P4. The colors correspond to the tracers considered in this paper and the dots are the average value.

if an observer is not surrounded by a bubble (i.e., a low-density cavity), then nearby molecular clouds may completely dominate the tracer signal seen by that observer over large patches of the sky. This situation might prevent any detection of spiral arms.

As outlined in Sect. 2.2 we forced the creation of bubbles into the spiral arms at distinct positions by adjusting the local SN parameters of the MHD simulation. The emerging bubbles are approximately $8 \mathrm{kpc}$ from the Galactic center and roughly 50-300 pc wide in order to provide conditions comparable to the Local Bubble surrounding our own solar neighborhood (Fuchs et al. 2009; Liu et al. 2017; Alves et al. 2018). We place the observer at the four distinct positions labeled P1, P2, P3, and P4 (see Fig. 2). Each position represents a different stage of the bubble evolution.

Position $\mathrm{P} 1$ is surrounded by a cavity with a central gas density of $n_{\mathrm{g}} \approx 0.1 \mathrm{~cm}^{-3}$, an extension of roughly $100-150 \mathrm{pc}$, and is the most similar to our Local Bubble (Fuchs et al. 2009; Liu et al. 2017; Alves et al. 2018). P1 is in a semi-static stage between the expansion and collapse phase. Consequently, the velocity field and the magnetic field lines are well ordered. The resulting profiles have peaks above the noise level and the $\mathrm{CO}$ velocity has well-defined loops (see Figs. 3 and 5), which allow the detection of tangent points.

In Fig. 10 we show the number of spiral arm detections for each observer position. Here we only count the detections of the points $\mathrm{C}-\mathrm{H}$ that are clearly separated from the blurry disk in the center of the MHD simulation. With the exception of synchrotron, all tracers can recover the tangent points $\mathrm{C}-\mathrm{H}$. We calculate the relative deviations for each tracer and tangent angle point with respect to the $\mathrm{CO}$ data. We plot the maximum and minimum deviation of all tangent points in Fig. 10. For P1, the maximum deviation to the $\mathrm{CO}$ angle is $-6.88^{\circ}$. We report a systematic offset of roughly $-2^{\circ}$ for all tracers. Hou \& Han (2015) found the offsets between gaseous tracers of spiral arm tangents to be of this magnitude. We speculate that this systematic offset may also be related to the offset for the tracers of synchrotron, dust, and RM discussed in Sect. 5.3.

Position P2 is already in its collapse phase, where the cavity is filling up with gas. The local density is almost the same as in the local arm of P2. The velocity field and gas density distribution are much more turbulent than at the location of P1.
The loops of the local arm are broken in the longitude-velocity diagram and one of the local arm tangents cannot be detected. This also affects the synchrotron emission and the dust signal. Due to the increase in noise, some of the tangent points can no longer be detected. The only tracer that allows us to recover all six tangent points is the RM. Here the range of deviations from the $\mathrm{CO}$ tangents is much larger compared to P1 with angles up to $9.85^{\circ}$. Only the synchrotron detections seem to agree with CO. However, for P2 the synchrotron detection is limited and shows detectable signs of only two of the six tangent points. We note that the low number of detections in the synchrotron signal may be biased by our simple distribution of CR electrons For actual detections of tangents within the Milky Way synchrotron observations may still be tracers of tangents.

The bubble embedding P3 has just recently been formed and is still expanding. Hence, the magnetic field is increased compared to the $\mathrm{P} 1$ and $\mathrm{P} 2$ positions and the gas surrounding the bubble is highly turbulent. The bubble itself is almost completely evacuated with a density on the order of $n_{\mathrm{g}} \approx 10^{-4} \mathrm{~cm}^{-3}$ and a maximum extension of about 300 pc. P3 is also not that close to the spine of a spiral arm, but located in a diffuse interarm region. Despite these difficulties all tangent points are detectable with CO observations, whereas all other tracers are missing some tangent point detection. Here, with values up to $-11.42^{\circ}$ the deviations from the detected angles by $\mathrm{CO}$ are even larger compared to those of $\mathrm{P} 1$ and $\mathrm{P} 2$.

Finally, the observer at P4 is placed directly into a spiral arm unaffected by any SN feedback. Hence, the gas density is very smooth with well-behaved magnetic field lines and a regular velocity field. For P4 the longitude-velocity diagram shows a broad luminous band within $\pm 25 \mathrm{~km} \mathrm{~s}^{-1}$ emerging from the local environment. This band prevents the detection of the tangent points of the local arm of $\mathrm{P} 4$. The remaining arms $\mathrm{C}$, $\mathrm{F}, \mathrm{G}$, and $\mathrm{H}$ are clearly separated in velocity space. Dust and synchrotron emission are also heavily influenced by features of nearby clouds, and characteristic peaks related to any tangent points become buried in noise. Only the RM allows to detect all tangents. We speculate that this may be due to the smooth electron fraction and magnetic field surrounding position $\mathrm{P} 4$. The deviations of the tangent angles with values up to $-10.38^{\circ}$ are generally smaller than those of P2 and P3. 
One may intuitively expect that an inner arm location without a bubble may provide the worst environment for observing one's own galaxy. However, as Fig. 10 reveals, the turbulence of gas and magnetic field fluctuations also seem to be important factors. In essence, the conditions of P1 represent the most ideal environment for spiral arm detections within the set of observer positions considered in this paper.

\section{Caveats}

There are several limitations in our Milky Way simulation that potentially have an impact on the synthetic observations presented in this paper. First, the cosmic ray ionization rate of $\zeta_{\mathrm{H}}=3 \times 10^{-17} \mathrm{~s}^{-1}$ adopted in the simulations of Smith et al. (2020) and Chen et al. (in prep.) is somewhat lower than the values indicated by observations of $\mathrm{H}_{3}^{+}, \mathrm{OH}^{+}, \mathrm{H}_{2} \mathrm{O}^{+}$, and $\mathrm{ArH}^{+}$ in the diffuse ISM (Indriolo \& McCall 2012; Indriolo et al. 2015; Neufeld \& Wolfire 2017), which are more consistent with a value an order of magnitude higher. Therefore, the simulations will tend to underestimate the electron density in regions where cosmic ray ionization dominates by a factor of $\sqrt{10} \sim 3$. Since $\mathrm{RM} \propto n_{\mathrm{el}}$, this means that we may underestimate the actual RM values by a factor of a few. We note, however, that this will only affect the overall normalization of our RM values and not their relative magnitude as a function of 1 . Therefore, this uncertainty should have no effect on the structure we see in the RM profiles.

Second, as our simulation does not account for photoionization feedback from massive stars, it will miss any contribution from ionized gas within and on the boundaries of HII regions to the measured RM values. We should therefore not expect good agreement between our results and observations of RM toward known Galactic HII regions (see, e.g., Purcell et al. 2015). However, the high values of RM found by Shanahan et al. (2019) of around $1=48^{\circ}$ are largely found away from HII regions, and hence it is meaningful to compare these with our simulation results.

Third, our neglect of radiative feedback from massive stars also means that we will underestimate the dust temperature and hence the $870 \mu \mathrm{m}$ continuum flux in the vicinity of young stellar clusters. However, this should not have a large impact on our results. At $870 \mu \mathrm{m}$, we are in the Rayleigh-Jeans tail of the dust emission spectrum and so the brightness of the emission varies only slightly faster than linearly with the dust temperature. Since the dust temperature varies with the dust heating rate $\Gamma_{\text {dust }}$ as $T_{\mathrm{d}} \propto \Gamma_{\text {dust }}^{\alpha}$ with $\alpha=1 / 5-1 / 6$, depending on the dust properties, even large spatial variations in the dust heating rate introduce only small variations into the dust temperature and the $870 \mu \mathrm{m}$ flux.

Finally, our model of CO formation and destruction is highly approximate and has been shown by Glover \& Clark (2012) to overproduce $\mathrm{CO}$ in molecular cloud conditions. In addition, even though the spatial resolution of the Chen et al. (in prep). simulations is excellent by the standards of simulations of entire galactic disks ${ }^{7}$, it is not sufficient to yield a completely numerically converged CO distribution (Joshi et al. 2019). We would therefore expect some of the small-scale details of the $\mathrm{CO}$ distribution to change if we increased the numerical resolution of the simulation or adopted a more accurate treatment of the $\mathrm{CO}$ chemistry. However, the large-scale distribution of $\mathrm{CO}$ emission in position-velocity space should remain largely unaffected, and

\footnotetext{
7 Compare, for instance, the Chen et al. (in prep.) resolution of $\sim 1 \mathrm{pc}$ at $n=100 \mathrm{~cm}^{-3}$ with the values at a similar resolution of $8 \mathrm{pc}$ in Fujimoto et al. (2019) or $\sim 20$ pc in Körtgen et al. (2019).
}

so we would not expect the results presented in this paper to be sensitive to our choice of chemical model or our numerical resolution in dense regions.

\section{Summary and conclusions}

We present a comparison of synthetic spiral arm detections and observations of the Milky Way within the Galactic plane. Synthetic observations are created based on a MHD simulation of the Galactic disk. The simulation provides a Milky Way-like spiral structure and includes gas self-gravity, chemistry, and SN feedback. In a post-processing step we synthesize spiral arm observations by mimicking the RT physics of cold dust emission, synchrotron radiation, line emission, and Faraday rotation. We analyze the synthetic longitudinal profiles for each individual tracer in order to detect the characteristic signatures of spiral arm tangents. This physical model for Faraday rotation in the Milky Way is compared with Faraday rotation from the THOR survey to investigate the contribution of spiral arms to the total rotation measure of the Milky Way.

- We report an angular separation of about $\pm 6^{\circ}$ of tangent points observed in different tracers. This separation is similar to that of the Milky Way.

- Our models confirm that the pitch angle of the Milky Way's spiral arms may be recovered from its tangent points on the basis of trigonometrical considerations. The recovered range of angles in our models is in agreement with the pitch angles observed in the Milky Way.

- The RM signal is traced through the simulations along distinct LOSs as it accumulates on its way toward the observer. Here we find that spiral arms create characteristic peaks in Galactic Faraday rotation with contrast and longitude extent that depend on viewing geometry and galactocentric radius of the tangent point. We offer an explanation of this profile based on a simple geometrical model of a spiral galaxy.

- We find characteristic peaks in the RM signal observed in the Milky Way that may be attributed to the Local Arm (Orion spur) where our Solar System is situated. The pitch angle corresponding to these peaks agrees with the angles given in the literature. Hence, this finding provides an additional means to validate the orientation of the Orion spur within the Milky Way.

- The pattern of a sharp peak in Faraday rotation flanked by low RM on the outside, and elevated RM on the inside as reported by Shanahan et al. (2019) is reproduced by the simulations. Other spiral arms may show a broader peak depending on the distance of the tangent point from the observer and from the Galactic center.

- We repeat our analysis for observers situated in distinct SN bubbles. These bubbles represent a density cavity in different stages of their development within the MHD spiral arms. Our analysis reveals that a local bubble most similar to our own Solar neighborhood provides the best Galactic environment for the detection of spiral arm tangents.

The analysis of the resulting synthetic observations lays the groundwork for the interpretation of current and future observations of the Milky Way and their comparison with the theory of galaxy evolution.

Acknowledgements. Special thanks go to Claire Rajkay for useful discussions. S.R., S.C.O.G., R.S.K., M.R.R. and H.B. acknowledge funding from the Deutsche Forschungsgemeinschaft (DFG, German Research Foundation) Project-ID 138713538 - SFB 881 "The Milky Way System" (sub-projects A06, B01, B02, and B08) and from the Priority Program SPP 1573 "Physics of the Interstellar Medium" (grant numbers KL 1358/18.1, KL 1358/19.2). S.R., 
S.C.O.G. and R.S.K. acknowledge support from the DFG via the Heidelberg Cluster of Excellence STRUCTURES in the framework of Germany's Excellence Strategy (grant EXC-2181/1 - 390900948). The authors gratefully acknowledge the data storage service SDS@hd supported by the Ministry of Science, Research and the Arts Baden-Württemberg (MWK) and the German Research Foundation (DFG) through grant INST 35/1314-1 FUGG and support by the state of Baden-Württemberg through bwHPC and the German Research Foundation (DFG) through grant INST 35/1134-1 FUGG. J.M.S acknowledges the support of the Natural Sciences and Engineering Research Council of Canada (NSERC), 2019-04848. R.J.S. acknowledges an STFC Ernest Rutherford fellowship(grant ST/N00485X/1)and HPC from the Durham DiRAC super-computing facility. H.B. and J.D.S. acknowledge support from the European Research Council under the Horizon 2020 Framework Program via the ERC Consolidator Grant CSF-648505.

\section{References}

Alves, M. I. R., Boulanger, F., Ferrière, K., \& Montier, L. 2018, A\&A, 611, L5 Beck, R. 2001, Space Sci. Rev., 99, 243

Beck, R. 2015, A\&ARv, 24, 4

Beck, R., \& Wielebinski, R. 2013, Planets, Stars and Stellar Systems, eds. T. D. Oswalt, \& G. Gilmore (Berlin: Springer), 5, 641

Bennett, C. L., Hill, R. S., Hinshaw, G., et al. 2003, ApJS, 148, 97

Berkhuisen, E. M., R., B., \& Hoernes, P. 2003, A\&A, 398, 937

Beuermann, K., Kanbach, G., \& Berkhuijsen, E. M. 1985, A\&A, 153, 17

Beuther, H., Tackenberg, J., Linz, H., et al. 2012, ApJ, 747, 43

Beuther, H., Bihr, S., Rugel, M., et al. 2016, A\&A, 595, A32

Bjorkman, J. E., \& Wood, K. 2001, ApJ, 554, 615

Bovy, J., Allende Prieto, C., Beers, T. C., et al. 2012, ApJ, 759, 131

Brauer, R., Wolf, S., \& Flock, M. 2017a, A\&A, 607, A104

Brauer, R., Wolf, S., Reissl, S., \& Ober, F. 2017b, A\&A, 601, A90

Burn, B. J. 1966, MNRAS, 133, 67

Chen, B. Q., Huang, Y., Hou, L. G., et al. 2019, MNRAS, 487, 1400

Clark, P. C., Glover, S. C. O., \& Klessen, R. S. 2012, MNRAS, 420, 745

Cordes, J. M., \& Lazio, T. J. W. 2002, ArXiv e-prints [arXiv:astro-ph/0207156]

Cox, D. P., \& Gomez, G. C. 2002, ApJS, 142, 261

Dame, T. M., Ungerechts, H., Cohen, R. S., et al. 1987, ApJ, 322, 706

Dame, T. M., Hartmann, D., \& Thaddeus, P. 2001, ApJ, 547, 792

Dohnanyi, J. S. 1969, J. Geophys. Res., 74, 2531

Draine, B. T. 1978, ApJS, 36, 595

Drimmel, R. 2000, A\&A, 358, L13

Drimmel, R., \& Spergel, D. N. 2001, ApJ, 556, 18

Ferriere, K. M., Mac Low, M.-M., \& G., Z. E. 1991, ApJ, 375, 239

Fletcher, A., Beck, R., Shukurov, A., Berkhuijsen, E. M., \& Horellou, C. 2011, MNRAS, 412, 2396

Flower, D. R. 2001, J. Phys. B, 34, 2731

Frick, P., Stepanov, R., Beck, R., et al. 2016, A\&A, 585, A21

Fuchs, B., Breitschwerdt, D., de Avillez, M. A., \& Dettbarn, C. 2009, Space Sci. Rev., 143, 437

Fujimoto, Y., Chevance, M., Haydon, D. T., Krumholz, M. R., \& Kruijssen, J. M. D. 2019, MNRAS, 487, 1717

Gaensler, B. M., Haverkorn, M., Staveley-Smith, L., et al. 2005, Science, 307, 1610

Glover, S. C., \& Clark, P. C. 2012, MNRAS, 421, 116

Glover, S. C. O., \& Mac Low, M.-M. 2007, ApJS, 169, 239

Górski, K. M., Hivon, E., Banday, A. J., et al. 2005, ApJ, 622, 759

Grand, R. J. J., Gómez, F. A., Marinacci, F., et al. 2017, MNRAS, 467, 179

Han, J. L., Manchester, R. N., Lyne, A. G., Qiao, G. J., \& van Straten, W. 2006, ApJ, 642, 868

Haslam, C. G. T., Klein, U., Salter, C. J., et al. 1981, A\&A, 100, 209

Haslam, C. G. T., Salter, C. J., Stoffel, H., \& Wilson, W. E. 1982, A\&AS, 47, 1

Heyer, M., Krawczyk, C., Duval, J., \& Jackson, J. M. 2009, ApJ, 699, 1092

Hou, L. G., \& Han, J. L. 2014, A\&A, 569, A125

Hou, L. G., \& Han, J. L. 2015, MNRAS, 454, 626

Huang, L., \& Shcherbakov, R. V. 2011, MNRAS, 416, 2574

Hutschenreuter, S., \& Enßlin, T. A. 2020, A\&A 633, A15

Indriolo, N., \& McCall, B. J. 2012, ApJ, 745, 91

Indriolo, N., Neufeld, D. A., Gerin, M., et al. 2015, ApJ, 800, 40

Inoue, T., Hennebelle, P., Fukui, Y., et al. 2018, PASJ, 70, S53

Jackson, J. M., Rathborne, J. M., Shah, R. Y., et al. 2006, ApJS, 163, 145

Jankowski, P., \& Szalewicz, K. 2005, J. Chem. Phys., 123, 104301

Jansson, R., \& Farrar, G. R. 2012, ApJ, 757, 14

Jeans, J. H. 1902, Phil. Trans. R. Soc. London Ser. A, 199, 1
Joshi, P. R., Walch, S., Seifried, D., et al. 2019, MNRAS, 484, 1735 Klessen, R. S., \& Glover, S. C. 2016, Saas Fee Lecture Notes, 43, 85 Körtgen, B., Banerjee, R., Pudritz, R. E., \& Schmidt, W. 2018, MNRAS, 479 L40

Körtgen, B., Banerjee, R., Pudritz, R. E., \& Schmidt, W. 2019, MNRAS, 489, 5004

Krumholz, M. R., \& Tan, J. C. 2007, ApJ, 654, 304

Langer, W. D., Velusamy, T., Goldsmith, P. F., et al. 2017, A\&A, 607, A59

Liu, W., Chiao, M., Collier, M. R., et al. 2017, ApJ, 834, 33

Lucy, L. B. 1999, A\&A, 344, 282

Mathis, Rumpl, \& Nordsieck 1977, ApJ, 217, 425

McMillan, P. J. 2017, MNRAS, 465, 76

Miville-Deschênes, M.-A., Ysard, N., Lavabre, A., et al. 2008, A\&A, 490, 1093

Mora-Partiarroyo, S. C., Krause, M., Basu, A., et al. 2019, A\&A, 632, A21

Nelson, R. P., \& Langer, W. D. 1997, ApJ, 482, 796

Neufeld, D. A., \& Wolfire, M. G. 2017, ApJ, 845, 163

Ober, F., Wolf, S., Uribe, A. L., \& Klahr, H. H. 2015, A\&A, 579, A105

Oppermann, N., Junklewitz, H., Robbers, G., et al. 2012, A\&A, 542, A93

Ossenkopf, V. 1997, New Astron., 2, 365

Pakmor, R., Gomez, F. A., Grand, R. J. J., et al. 2017, MNRAS, 16, 1

Pakmor, R., Guillet, T., Pfrommer, C., et al. 2018, MNRAS, 481, 4410

Pakmor, R., \& Springel, V. 2013, MNRAS, 432, 176

Pandya, A., Zhang, Z., Chandra, M., \& Gammie, C. F. 2016, ApJ, 822, 34

Pellegrini, E. W., Reissl, S., Rahner, D., et al. 2020, MNRAS, 498, 3193

Planck Collaboration XII. 2020, A\&A, 641, A12

Planck Collaboration Int. XIX. 2015, A\&A, 576, A104

Powell, K. G., Roe, P. L., Linde, T. J., Gombosi, T. I., \& Zeeuw, D. L. 1999, J. Comp. Phys., 154, 284

Purcell, C. R., Gaensler, B. M., Sun, X. H., et al. 2015, ApJ, 804, 22

Ragan, S. E., Moore, T. J. T., Eden, D. J., et al. 2018, MNRAS, 479, 2361

Reid, M. J., Menten, K. M., Brunthaler, A., et al. 2014, ApJ, 783, 130

Reid, M. J., Menten, K. M., Brunthaler, A., Zheng, X. W., \& Dame, T. M. 2019, ApJ, 885, 131

Reissl, S., Wolf, S., \& Brauer, R. 2016, A\&A, 593, A87

Reissl, S., Seifried, D., Wolf, S., Banerjee, R., \& Klessen, R. S. 2017, A\&A, 603, A71

Reissl, S., Stutz, A. M., Brauer, R., et al. 2018, MNRAS, 481, 2507

Reissl, S., Brauer, R., Klessen, R. S., \& Pellegrini, E. W. 2019, ApJ, 885, 15

Roberts, W. W. 1969, ApJ, 158, 123

Rybicki, G. B., \& Lightman, A. P. 1979, Radiative Processes in Astrophysics (Weinheim: Wiley-VCH)

Schnitzeler, D. H. F. M., Carretti, E., Wieringa, M. H., et al. 2019, MNRAS, 485, 1293

Schöier, F. L., van der Tak, F. F. S., van Dishoeck, E. F., \& Black, J. H. 2005, A\&A, 432, 369

Shanahan, R., Lemmer, S. J., Stil, J. M., et al. 2019, ApJ, 887, L7

Shetty, R., Glover, S. C., Dullemond, C. P., et al. 2011, MNRAS, 415, 3253

Simard-Normandin, M., \& Kronberg, P. P. 1979, Nature, 279, 115

Smith, V. V., Hinkle, K. H., Cunha, K., et al. 2002, AJ, 124, 3241

Smith, R. J., Glover, S. C. O., Clark, P. C., Klessen, R. S., \& Springel, V. 2014 MNRAS, 1645, 1628

Smith, R. J., Treß, R. G., Sormani, M. C., et al. 2020, MNRAS, 492, 1594

Sokoloff, D. D., Bykov, A. A., Shukurov, A., et al. 1998, MNRAS, 299, 189

Sormani, M. C., Binney, J., \& Magorrian, J. 2015a, MNRAS, 454, 1818

Sormani, M. C., Binney, J., \& Magorrian, J. 2015b, MNRAS, 449, 2421

Sormani, M. C., Sobacchi, E., Shore, S. N., Treß, R. G., \& Klessen, R. S. 2017 MNRAS, 471, 2932

Sormani, M. C., Treß, R. G., Ridley, M., et al. 2018, MNRAS, 475, 2383

Springel, V. 2010, MNRAS, 401, 791

Sun, X. H., Reich, W., Waelkens, A., \& Enßlin, T. A. 2008, A\&A, 477, 573

Taylor, J. H., \& Cordes, J. M. 1993, ApJ, 411, 674

Taylor, A. R., Stil, J. M., \& Sunstrum, C. 2009, ApJ, 702, 1230

Tomisaka, K. 1998, MNRAS, 298, 797

Tress, R. G., Smith, R. J., Sormani, M. C., et al. 2020, MNRAS, 492, 2973

Truelove, J. K., Klein, R. I., McKee, C. F., et al. 1997, ApJ, 489, L179

Vallée, J. P. 2015, MNRAS, 450, 4277

Walch, S., Girichidis, P., Naab, T., et al. 2015, MNRAS, 454, 238

Wang, Y., Beuther, H., Rugel, M. R., et al. 2020, A\&A, 634, A83

Webber, W. R. 1998, ApJ, 506, 329

Weingartner, J. C., \& Draine, B. T. 2001, ApJ, 548, 296

Wernli, M., Valiron, P., Faure, A., et al. 2006, A\&A, 446, 367

Yao, J. M., Manchester, R. N., \& Wang, N. 2017, ApJ, 835, 29 